\title{
Hysteresis and bistability in the direct transition from 1:1 to $2: 1$ rhythm in periodically driven single ventricular cells
}

\author{
Ali R. Yehia, Dominique Jeandupeux, Francisco Alonso, and Michael R. Guevara ${ }^{\mathrm{a})}$ \\ Department of Physiology and Centre for Nonlinear Dynamics in Physiology and Medicine, \\ McGill University, Montréal, Québec H3G 1Y6, Canada
}

(Received 18 June 1999; accepted for publication 1 September 1999)

\begin{abstract}
The transmembrane potential of a single quiescent cell isolated from rabbit ventricular muscle was recorded using a suction electrode in whole-cell recording mode. The cell was then driven with a periodic train of current pulses injected into the cell through the same recording electrode. When the interpulse interval or basic cycle length (BCL) was sufficiently long, 1:1 rhythm resulted, with each stimulus pulse producing an action potential. Gradual decrease in BCL invariably resulted in loss of 1:1 synchronization at some point. When the pulse amplitude was set to a fixed low level and BCL gradually decreased, $N+1: N$ rhythms $(N \geqslant 2)$ reminiscent of clinically observed Wenckebach rhythms were seen. Further decrease in BCL then yielded a 2:1 rhythm. In contrast, when the pulse amplitude was set to a fixed high level, a period-doubled 2:2 rhythm resembling alternans rhythm was seen before a 2:1 rhythm occurred. With the pulse amplitude set to an intermediate level (i.e., to a level between those at which Wenckebach and alternans rhythms were seen), there was a direct transition from 1:1 to 2:1 rhythm as the BCL was decreased: Wenckebach and alternans rhythms were not seen. When at that point the BCL was increased, the transition back to 1:1 rhythm occurred at a longer BCL than that at which the $\{1: 1 \rightarrow 2: 1\}$ transition had initially occurred, demonstrating hysteresis. With the BCL set to a value within the hysteresis range, injection of a single well-timed extrastimulus converted 1:1 rhythm into 2:1 rhythm or vice versa, providing incontrovertible evidence of bistability (the coexistence of two different periodic rhythms at a fixed set of stimulation parameters). Hysteresis between 1:1 and 2:1 rhythms was also seen when the stimulus amplitude, rather than the BCL, was changed. Simulations using numerical integration of an ionic model of a single ventricular cell formulated as a nonlinear system of differential equations provided results that were very similar to those found in the experiments. The steady-state action potential duration restitution curve, which is a plot of the duration of the action potential during 1:1 rhythm as a function of the recovery time or diastolic interval immediately preceding that action potential, was determined. Iteration of a finite-difference equation derived using the restitution curve predicted the direct $\{1: 1 \leftrightarrow 2: 1\}$ transition, as well as bistability, in both the experimental and modeling work. However, prediction of the action potential duration during 2:1 rhythm was not as accurate in the experiments as in the model. Finally, we point out a few implications of our findings for cardiac arrhythmias (e.g., Mobitz type II block, ischemic alternans). (C) 1999 American Institute of Physics. [S1054-1500(99)01104-0]
\end{abstract}

The majority of cells in the heart are not spontaneously active. Instead, these cells are excitable, being driven into activity by periodic stimulation originating in a specialized pacemaker region of the heart containing spontaneously active cells. This pacemaker region normally imposes a 1:1 rhythm on the intrinsically quiescent cells. However, the 1:1 response can be lost when the excitability of the paced cells is decreased, when there are problems in the conduction of electrical activity from cell to cell, or when the heart rate is raised. When 1:1 synchronization is lost in the intact heart, one of a variety of abnormal cardiac arrhythmias can arise. In single quies-

\footnotetext{
a) Author to whom correspondence should be addressed. Department of Physiology, McGill University, 3655 Drummond Street, Room 1018, Montréal, Québec H3G 1Y6, Canada. Telephone: 514-398-4320; Fax: 514-3987452. Electronic mail: guevara@physio.mcgill.ca
}

cent cells isolated from ventricular muscle, 1:1 rhythm can be replaced by a $N+1: N$ rhythm $(N \geqslant 2)$, a perioddoubled 2:2 rhythm, or a 2:1 rhythm. We investigate below the direct transition from 1:1 to $2: 1$ rhythm in experiments on single cells and in numerical simulations of an ionic model of a single cell formulated as a nonlinear system of differential equations. We show that there is hysteresis associated with this transition in both model and experiment, and develop a theory for the bistability underlying this hysteresis that involves the coexistence of two stable fixed-points on a two-branched onedimensional map.

\section{INTRODUCTION}

During normal sinus rhythm, there is 1:1 synchronization between the natural pacemaker of the heart, the sinoatrial node, and the ventricles: i.e., any particular ventricu- 
lar cell is activated at a relatively fixed latency following activation of the sinoatrial node. When the ability of ventricular muscle to conduct the action potential is sufficiently compromised (by, e.g., ischemia, hyperkalemia), this 1:1 rhythm is lost and replaced by some new rhythm, such as Wenckebach block, ${ }^{1,2} 2: 1$ block, $^{3,4}$ alternans rhythm, ${ }^{3-11}$ or even complete block. ${ }^{3}$ Alternatively, if one paces healthy multicellular ventricular muscle at a fast enough rate, one can also obtain 2:1 rhythm ${ }^{12-14}$ or alternans rhythm. ${ }^{14-22}$ In Wenckebach block, there is an occasional skipping or dropping of an action potential that is typically preceded by a gradual beat-to-beat increase in the activation time of the immediately preceding beats; in 2:1 block, there is block of propagation of every second action potential; in alternans rhythm, there is a beat-to-beat alternation in the morphology of the action potential or of the propagation time; and in complete block, there is either a subthreshold response or no response at all detected at the recording site.

All of the above references deal with work carried out in multicellular preparations. In any such preparation, there are unavoidably intrinsic spatial factors that make it hard to determine the mechanisms underlying a particular rhythm. For example, in the ischemic ventricle, there are several reports that alternans in the action potential morphology is seen at sites that are just adjacent to areas displaying 2:1 block (see, e.g., Fig. 9 of Ref. 3; Fig. 5 of Ref. 4). The alternans is then quite possibly due to the fact that the input current to the cell under study is itself alternating from beat to beat, due to electrotonic coupling with the nearby site displaying 2:1 block. Should that be the case, the alternans cannot be said to be intrinsically generated by the cell from which it is recorded. Sorting out the mechanisms of alternans in inhomogeneous multicellular preparations will, at a minimum, require recording the transmembrane potential from many sites and also somehow measuring or estimating intercellular flows of current.

We, therefore, decided to first approach the simpler problem of what happens when 1:1 rhythm is lost in a single isolated ventricular cell when either the pacing frequency is increased or the stimulus amplitude is lowered. Both Wenckebach and alternans rhythms, which have been known for over a century in the intact heart, have been more recently described in isolated ventricular cells, with the former occurring at a considerably lower stimulus amplitude than the latter. In the former case one sees the transition $\{1: 1 \rightarrow$ Wenckebach $\rightarrow 2: 1\}$ as the pacing frequency is increased ${ }^{23-25}$ or the stimulus amplitude is decreased, ${ }^{26}$ while in the latter one sees the transition $\{1: 1 \rightarrow$ alternans $\rightarrow 2: 1\}$ as the pacing frequency is increased. ${ }^{25,27,28}$ Thus, other rhythms are encountered in the transition from 1:1 to 2:1 rhythm when the stimulus amplitude is either high or low. We report below that the transition from 1:1 to 2:1 rhythm can be direct (i.e., no other rhythms seen) in single rabbit ventricular cells when the stimulus amplitude is intermediate to those at which Wenckebach and alternans rhythms are seen.

\section{METHODS}

\section{A. Experimental techniques}

Single ventricular cells were enzymatically isolated from New Zealand white rabbits following previously published techniques. ${ }^{24,27}$ Procedures involving animals were approved by the University's Animal Care Committee and conformed to the requirements of the Canadian Council on Animal Care. Aliquots of the single-cell suspension were transferred to a superfusion chamber mounted on the stage of an inverted microscope $\left(22-24^{\circ} \mathrm{C}\right)$. An amplifier (Axoclamp 2A, Axon Instruments, Foster City, CA), bridge-balanced and capacity compensated, was used in whole-cell recording mode to record the transmembrane potential and to inject current pulses. Signals were recorded on a video-cassette recorder after A/D conversion at 22 or $44 \mathrm{kHz}$ (Neuro-Corder DR484, Neuro Data Instruments Corp., New York, NY) for later off-line analysis. Data was then re-sampled at $1 \mathrm{kHz}$ (with appropriate anti-alias low-pass filtering at $500 \mathrm{~Hz}$ ) and displayed using the action potential capture and analysis program AP (Alembic Software, Montreal).

\section{B. Numerical techniques}

We employed the Luo \& Rudy (LR) model of spaceclamped ventricular membrane, ${ }^{29}$ which is formally an eightdimensional nonlinear ordinary differential equation. We implemented a simple integration scheme with a fixed integration time step $(\Delta t)$ of $0.001 \mathrm{~ms}$. The value of each activation or inactivation variable $\xi_{i}$ at time $t+\Delta t$ was obtained from its value at time $t$ using the analytic formula ${ }^{30}$

$$
\xi_{i}(t+\Delta t)=\xi_{i}(\infty)-\left[\xi_{i}(\infty)-\xi_{i}(t)\right] e^{-\Delta t / \tau_{i}},
$$

where $\xi_{i}(\infty)$ is the steady-state or asymptotic value of $\xi_{i}$ and $\tau_{i}$ is the time-constant of $\xi_{i}$, both of which are functions of the transmembrane potential $(V)$. A simple forward Euler scheme was used to obtain the internal calcium concentration $\left(\left[\mathrm{Ca}^{++}\right]_{i}\right)$

$$
\left[\mathrm{Ca}^{++}\right]_{i}(t+\Delta t)=\left[\mathrm{Ca}^{++}\right]_{i}(t)+\left(d\left[\mathrm{Ca}^{++}\right]_{i}(t) / d t\right) \Delta t .
$$

The external potassium concentration was set to the nominal LR value of $5.4 \mathrm{mM}$. As pointed out recently, ${ }^{31}$ there are certain internal inconsistencies within the Luo-Rudy article: In what follows, we use the equations appearing in Table 1 of Luo and Rudy (1991). ${ }^{29}$ The value of $R T / F(R=$ Rydberg gas constant, $T=$ temperature $\left({ }^{\circ} \mathrm{K}\right), F=$ Faraday constant), used in calculating reversal potentials, is not given in Ref. 29 . We use $R T / F=26.5 \mathrm{mV}$ in our program, which results in $E_{K}$ $\cong-76.95 \mathrm{mV}$ (vs value of $-77 \mathrm{mV}$ stated in Ref. 29) and $E_{\mathrm{Na}} \cong 54.35 \mathrm{mV}$ (vs $54.4 \mathrm{mV}$ in Ref. 29) at the nominal LR temperature of $37^{\circ} \mathrm{C}$. The maximum $\Delta V$ in our simulations in going from time $t$ to time $t+\Delta t$ (with $\Delta t=0.001 \mathrm{~ms}$ ) was $\sim 0.35 \mathrm{mV}$.

The currents in the LR model are expressed in $\mu \mathrm{A} / \mu \mathrm{F}$; in what follows, we have taken a nominal cell capacitance of $50 \mathrm{pF}$ to facilitate comparison with our experimental results. Our standard initial conditions were obtained by recording the values of the system variables after applying a conditioning series of 50 stimuli at a basic cycle length of $450 \mathrm{~ms}$ (pulse amplitude $=225 \mathrm{pA}$, pulse duration $=10 \mathrm{~ms}$ ) from the 
infinitely rested state. All programs were written in C (Microsoft Visual $\mathrm{C}++$, Microsoft Corp. Redmond, WA) using long floating point variables ( $\sim 14$ significant decimal digits).

\section{RESULTS}

\section{A. The direct $\{1: 1 \rightarrow 2: 1\}$ transition}

We stimulated a cell by delivering a train of fixed duration (range: 5-20 ms) current pulses at a fixed pulse amplitude and interpulse interval [basic cycle length (BCL)]. When both amplitude and BCL were sufficiently large, a cell generated an action potential of a fixed morphology for each injected pulse, resulting in a 1:1 rhythm. When BCL was gradually reduced, the cell at some point no longer responded in a 1:1 fashion. Previous systematic studies on spontaneously beating aggregates of embryonic chick ventricular cells have shown that there was eventually a transition to a 2:1 rhythm, and that this transition could either be directly from 1:1 rhythm or be interrupted by other rhythms (alternans or Wenckebach-type rhythms), depending on the exact magnitude of the pulse amplitude. ${ }^{32,33} \mathrm{We}$, therefore,
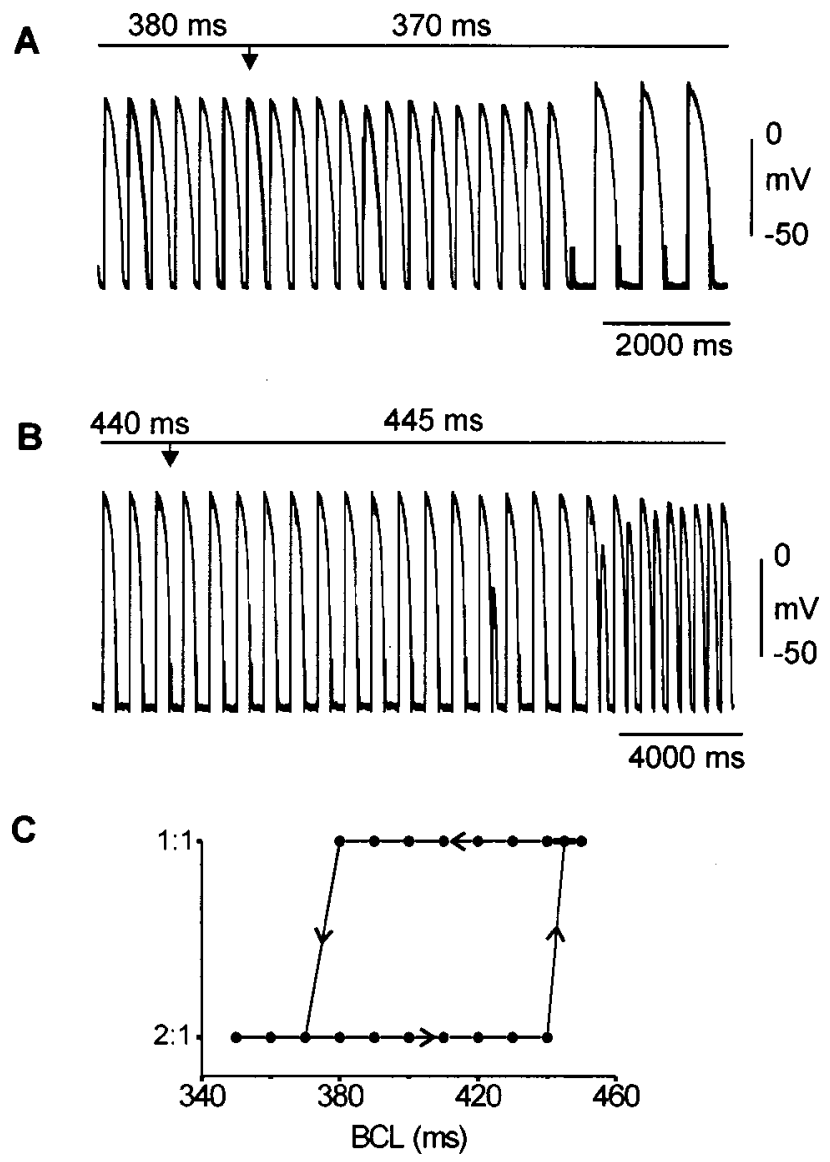

FIG. 1. Hysteresis in $\{1: 1 \leftrightarrow 2: 1\}$ transition (experiment). (A) $\{1: 1 \rightarrow 2: 1\}$ transition. Transmembrane potential plotted vs time. Arrow indicates beginning of first cycle with $\mathrm{BCL}=370 \mathrm{~ms}$. (B) $\{2: 1 \rightarrow 1: 1\}$ transition. Arrow indicates beginning of first cycle with $\mathrm{BCL}=445 \mathrm{~ms}$. (C) Hysteresis loop. Stimulus artefacts due to imperfect bridge balance retouched in (A) and (B) and all subsequent experimental traces by erasing part of the deflection: thus voltage during time of stimulus pulse injection is only an estimate made based on recordings from other cells with negligible stimulus artefact. Pulse amplitude $=850 \mathrm{pA}$, pulse duration $=5 \mathrm{~ms}$. scanned a range of pulse amplitudes for each cell, until we found the amplitude at which there was a direct transition from 1:1 to $2: 1$ rhythm.

At a pulse amplitude appropriate to obtaining the direct $\{1: 1 \rightarrow 2: 1\}$ transition, the BCL was gradually decreased in steps of $5,10,15$, or $20 \mathrm{~ms}$ from a value sufficiently high to generate 1:1 rhythm. At each BCL, stimulation was maintained for at least 20 stimuli to help dissipate any transients. As BCL was reduced, there was eventually a transition to $2: 1$ rhythm. Figure 1(A) shows an example of a direct transition from 1:1 to 2:1 rhythm using the above protocol. The cell was stimulated at a BCL of $450 \mathrm{~ms}$, pulse duration of $5 \mathrm{~ms}$, and pulse amplitude of $850 \mathrm{pA}$, which resulted in 1:1 rhythm (the threshold amplitude to obtain 1:1 rhythm at $\mathrm{BCL}=500$ ms was $\sim 800 \mathrm{pA}$ in this cell). The BCL was then lowered in decrements of $10 \mathrm{~ms}$. When the BCL was changed from 380 to $370 \mathrm{~ms}$ [arrow indicates beginning of first cycle at $\mathrm{BCL}$ $=370 \mathrm{~ms}$ in Fig. 1(A)], the 1:1 rhythm was replaced by a 2:1 rhythm, but only after a transient consisting of thirteen 1:1 cycles. During the transient, the amplitude of the action potentials decreased, while the beat-to-beat variability in amplitude increased. The exact form of this transient was highly variable from cell to cell, and even from trial to trial within the same cell.

The average stimulus amplitude at which the direct $\{1: 1 \rightarrow 2: 1\}$ transition was seen was $\sim 1.2$ times the threshold amplitude needed to obtain $1: 1$ rhythm at $\mathrm{BCL}=1000 \mathrm{~ms}$ $(n=12)$. In all cells in which the direct $\{1: 1 \rightarrow 2: 1\}$ transition was found, and in which an alternans or Wenckeback-like rhythm (e.g., 3:2 rhythm) was also encountered at a different pulse amplitude, alternans occurred at a pulse amplitude higher than that at which the $\{1: 1 \rightarrow 2: 1\}$ transition occurred (e.g., alternans was seen in the cell of Fig. 1 at an amplitude of $900 \mathrm{pA}$ ), while Wenckebach rhythm occurred at a lower pulse amplitude.

\section{B. Hysteresis between 1:1 and 2:1 rhythms}

Following the direct transition from 1:1 to 2:1 rhythm, further decrease in BCL resulted in 2:1 rhythm being maintained over a range of BCL. The BCL was then gradually increased. In the experiment of Fig. 1, upon increasing BCL from 440 to $445 \mathrm{~ms}$, the cell converted back from 2:1 to 1:1 rhythm [Fig. 1(B)]. Again, a transient was seen after the BCL was changed (arrow), this time consisting of several $2: 1$ cycles followed by several alternans cycles. As with the $\{1: 1 \rightarrow 2: 1\}$ transition, the transient was not the same from cell to cell or even from trial to trial, with the number of transient 2:1 cycles being between $\sim 2$ and $\sim 15$. The fact that the $\{2: 1 \rightarrow 1: 1\}$ transition occurred at a longer BCL from that at which the $\{1: 1 \rightarrow 2: 1\}$ transition had initially occurred demonstrates the existence of hysteresis. We shall refer to this hysteresis between 1:1 and 2:1 rhythms as " $\{1: 1 \leftrightarrow 2: 1\}$ hysteresis." Figure $1(\mathrm{C})$ shows the hysteresis loop, which was $\sim 75 \mathrm{~ms}$ wide in this cell. In each of five other cells in which the direct $\{1: 1 \rightarrow 2: 1\}$ transition was seen and in which a search for hysteresis was made, hysteresis was observed (hysteresis range $=25-100 \mathrm{~ms}, n=6$ ).

One possible explanation for hysteresis is that the elec- 
A

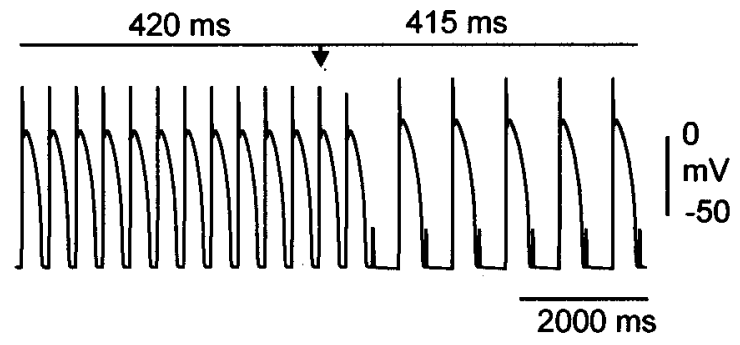

B

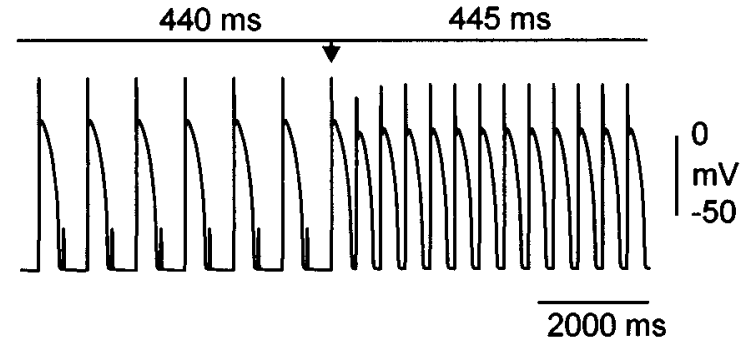

C

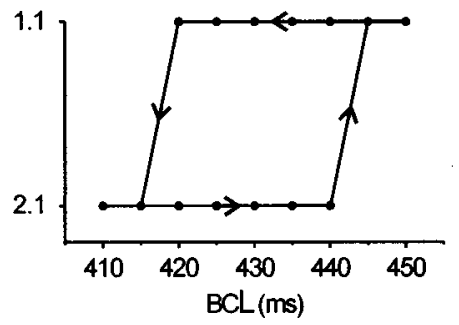

FIG. 2. Hysteresis in $\{1: 1 \leftrightarrow 2: 1\}$ transition (ionic model). (A) $\{1: 1 \rightarrow 2: 1\}$ transition. Arrow indicates beginning of first cycle at $\mathrm{BCL}=415 \mathrm{~ms}$. (B) $\{2: 1 \rightarrow 1: 1\}$ transition. Arrow indicates beginning of first cycle at $B C L=445$ ms. (C) Hysteresis loop. Pulse amplitude $=225 \mathrm{pA}$, pulse duration $=10 \mathrm{~ms}$.

trophysiological properties of the cell were slowly changing as the experiment proceeded, due to the well-known "rundown" of the calcium and other currents that occurs during recording with a patch-pipette in the whole-cell mode. ${ }^{34}$ Thus the inevitable delay between the times at which the two transitions shown in Figs. 1(A) and 1(B) occurred might put the cell into a different state. We tested the possibility that the hysteresis might be an artefact of rundown by making runs in which the BCL was first increased and then later decreased. We observed the same form of hysteresis (i.e., BCL shorter at the $\{1: 1 \rightarrow 2: 1\}$ transition than at the $\{2: 1 \rightarrow 1: 1\}$ transition), thus demonstrating that the hysteresis is a phenomenon independent of rundown.

Figure 2 gives the results of numerical simulations in the LR ionic model using the same type of stimulation protocol as in the experiments. Starting out with our standard initial conditions (approximate steady-state conditions appropriate to $\mathrm{BCL}=450 \mathrm{~ms}$, pulse amplitude $=225 \mathrm{pA}$, pulse duration $=10 \mathrm{~ms}$ ), we drove the model with an additional train of 20 stimuli at $\mathrm{BCL}=450 \mathrm{~ms}$ (threshold amplitude for 1:1 rhythm was $\sim 210 \mathrm{pA}$ at this BCL). The BCL was then decreased in steps of $5 \mathrm{~ms}$, injecting 20 stimuli at each BCL, until $\mathrm{BCL}=400 \mathrm{~ms}$ was reached. We then gradually increased the BCL in increments of $5 \mathrm{~ms}$ up to $\mathrm{BCL}=450 \mathrm{~ms}$, injecting 20 stimuli at each BCL. Upon lowering BCL from 420 to $415 \mathrm{~ms}$, there was a direct transition from 1:1 to $2: 1$ rhythm [Fig. 2(A)]. In contrast to the experimental data, the transition was much more immediate-following the change in BCL (arrow), there was only one transient 1:1 cycle. The transition from 2:1 to 1:1 rhythm occurred when BCL was increased from 440 to $445 \mathrm{~ms}$ [Fig. 2(B)]. Again in contrast to the experimental result, this transition was much more abrupt, with no transient 2:1 cycles being seen following the change in BCL (arrow). The hysteresis range in the model was $\sim 30 \mathrm{~ms}$ at this pulse amplitude [Fig. 2(C)].

Our recordings of the transmembrane potential in Figs. 1 and 2 lend support to the mechanisms put forth by Mines many years ago to explain the $\{1: 1 \leftrightarrow 2: 1\}$ hysteresis seen in the contractile activity of frog ventricle. ${ }^{12} \mathrm{We}$ now outline these mechanisms, as proposed by Mines. As BCL is gradually reduced, the BCL becomes so short that a stimulus eventually falls within the refractory period, producing a dropped beat [first dropped beat in Figs. 1(A) and 2(A)]. The next stimulus falls well beyond the end of the refractory period, so that an action potential results. However, because this beat had a much longer recovery time, its APD (action potential duration) is much longer than during the preceding 1:1 rhythm. In fact, the refractory period of this beat is now so long that the next beat is blocked, the cycle starts over, and 2:1 rhythm is established [Figs. 1(A) and 2(A)]. Since the APD and refractory period are now much longer than during 1:1 rhythm at the same BCL, in order to obtain the transition back to $1: 1$ rhythm, the BCL at this point must be increased to a value higher than that at which the $\{1: 1 \rightarrow 2: 1\}$ transition had originally occurred [Figs. 1(B) and 2(B)].

\section{Pulse-induced $\{1: 1 \rightarrow 2: 1\}$ flip}

The existence of hysteresis shows that at any given BCL within the hysteresis zone there is the co-existence of two stable periodic rhythms ("bistability"). This implies that the particular rhythm seen at that BCL depends on the state of the system at the point in time at which the BCL was changed to that particular value. This further implies that a suitably chosen perturbation should be able to flip the system from one rhythm to the other, ${ }^{12}$ with the perturbation taking the state-point of the system from the basin of attraction of the limit cycle generating one rhythm to the basin of attraction of the limit cycle generating the other rhythm.

The hysteresis experiment of Fig. 1 was performed with the cell initially being stimulated at a BCL of $500 \mathrm{~ms}$ (same pulse duration and amplitude as in Fig. 1), resulting in 1:1 rhythm. The BCL was then gradually dropped to $430 \mathrm{~ms}$, at which point 1:1 rhythm was still present. However, this BCL is within the hysteresis zone [Fig. 1(C)]. An extrastimulus pulse was then injected during the repolarizing phase of an action potential [arrow in Fig. 3(A)]. This extrastimulus prolonged the duration of that action potential just enough so that the next regularly scheduled stimulus fell within the refractory period, resulting in a skipped beat. This skipped beat was then followed by a very long recovery time, so that the next action potential had a much longer APD and refractory period than during the pre-existing 1:1 rhythm. Thus, the next stimulus produced a subthreshold response, and 2:1 rhythm was established. The perturbation thus flipped the rhythm directly and immediately from a $1: 1$ to a $2: 1$ rhythm (we shall refer to this effect as the " $\{1: 1 \rightarrow 2: 1\}$ flip"). The 


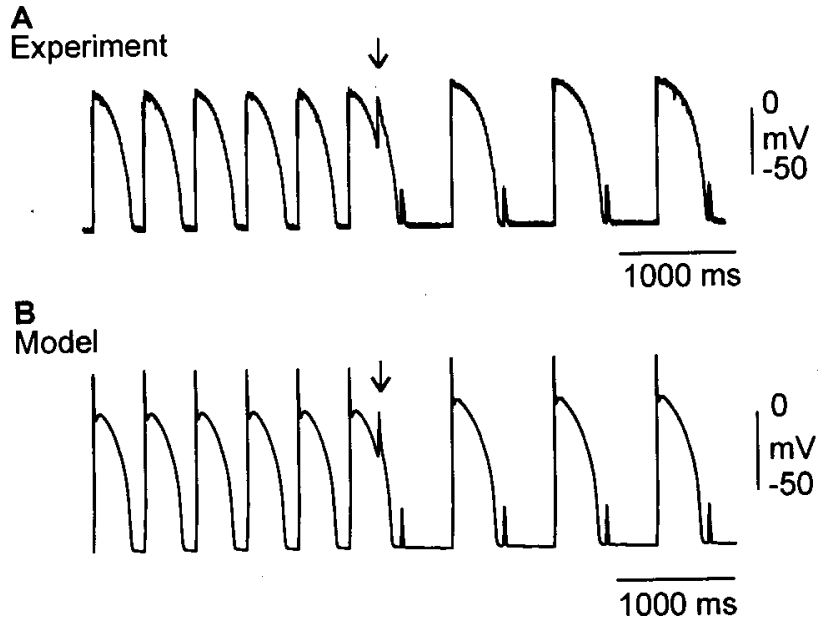

FIG. 3. $\{1: 1 \rightarrow 2: 1\}$ flip (experiment and model). (A) Experiment: BCL $=430$ $\mathrm{ms}$, pulse amplitude $=850 \mathrm{pA}$, pulse duration $=5 \mathrm{~ms}$. Same cell as in Fig. 1. (B) Model: $\mathrm{BCL}=430 \mathrm{~ms}$, pulse amplitude $=225 \mathrm{pA}$, pulse duration $=10$ ms. Coupling interval of extrastimulus $=250 \mathrm{~ms}$. Initial conditions for this run taken from the end of the twenty $1: 1$ cycles at $\mathrm{BCL}=430 \mathrm{~ms}$ in the hysteresis run of Fig. 2(C) (with BCL decreasing). Arrow indicates extrastimulus injection in (A) and (B).

timing of the pulse was critical, since pulses delivered at several different times earlier or later than the pulse shown in Fig. 3(A) did not produce the flip.

We next carried out the corresponding simulation of the $\{1: 1 \rightarrow 2: 1\}$ flip in the LR ionic model. During 1:1 rhythm at $\mathrm{BCL}=430 \mathrm{~ms}$, which is in the middle of the hysteresis zone [Fig. 2(C)], injection of an extrastimulus pulse [arrow in Fig. 3(B)] flipped the rhythm from 1:1 to $2: 1$. We next systematically investigated the effect of coupling interval (time from preceding action potential upstroke to pulse onset), changing it in steps of $10 \mathrm{~ms}$. As in the experiments, the timing was critical for the flip to occur: The extrastimulus had to be delivered with a coupling interval within the range 180-310 ms [e.g., Figs. 4(B) and 4(C)]. A stimulus that was delivered too early [10-170 ms, e.g., Fig. 4(A)] or too late

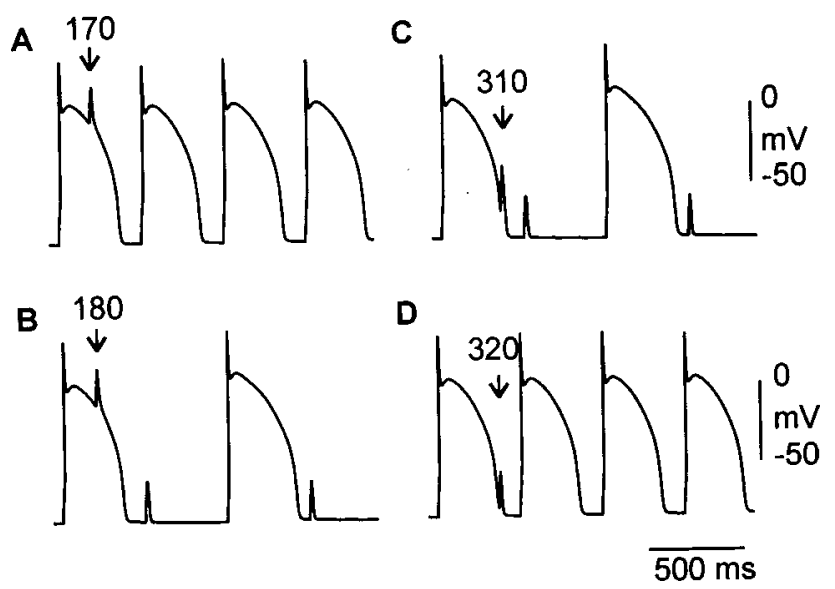

FIG. 4. Critical range of coupling intervals for $\{1: 1 \rightarrow 2: 1\}$ flip (model). The extrastimulus must fall within a critical intermediate range of coupling intervals to produce the $\{1: 1 \rightarrow 2: 1\}$ flip. Coupling interval=170 ms (A), 180 ms (B), $310 \mathrm{~ms}$ (C), $320 \mathrm{~ms}$ (D). Same stimulation parameters as in Fig. 3(B). These examples taken from a simulation in which the coupling interval was changed systematically between 10 and $420 \mathrm{~ms}$ in steps of $10 \mathrm{~ms}$.
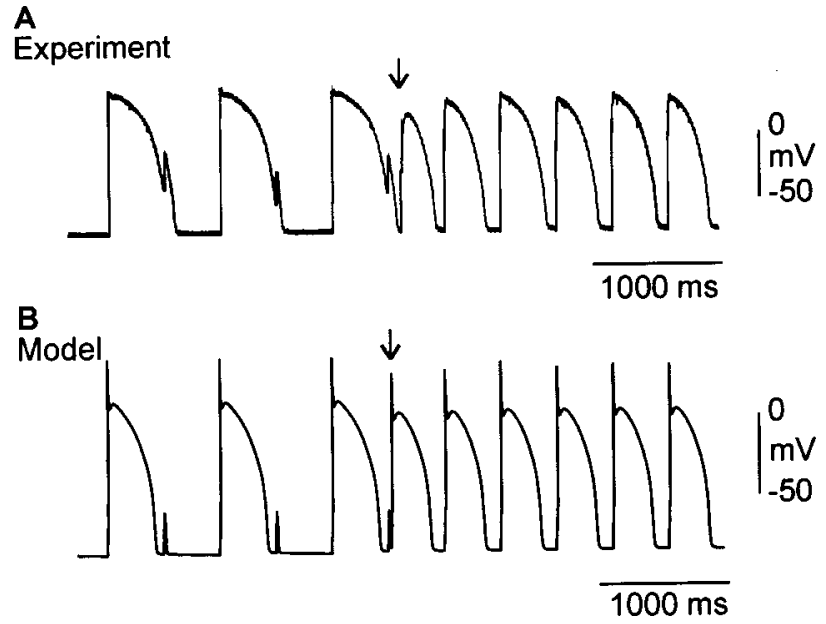

FIG. 5. $\{2: 1 \rightarrow 1: 1\}$ flip (experiment and model). (A) Experiment: $B C L=425$ $\mathrm{ms}$, pulse amplitude $=850 \mathrm{pA}$, pulse duration $=5 \mathrm{~ms}$. Same cell as in Figs. 1 and 3(A). (B) Model: $\mathrm{BCL}=430 \mathrm{~ms}$, pulse amplitude $=225 \mathrm{pA}$, pulse duration $=10 \mathrm{~ms}$. Initial conditions for this run taken from the end of the ten 2:1 cycles at $\mathrm{BCL}=430 \mathrm{~ms}$ in the hysteresis run of Fig. 2(C) (BCL increasing).

[320-420 ms, e.g., Fig. 4(D)] did not prolong the APD sufficiently to produce a skipped beat on the following stimulus, and so was incapable of precipitating the flip.

\section{Pulse-induced $\{2: 1 \rightarrow 1: 1\}$ flip}

In another run with the same cell as in Figs. 1 and 3(A), where 2:1 rhythm was seen at $\mathrm{BCL}=425 \mathrm{~ms}$ [Fig. 1(C)], injection of a single extrastimulus [arrow in Fig. 5(A)] very soon after a skipped beat produced an interpolated graded action potential and resulted in an immediate flip from 2:1 to 1:1 rhythm. We again carried out the corresponding simulation in the ionic model during $2: 1$ rhythm at $B C L=430 \mathrm{~ms}$, which is in the middle of the hysteresis zone [Fig. 2(C)]. Injection of an extrastimulus [arrow in Fig. 5(B)] produced an action potential, and flipped the rhythm from $2: 1$ to $1: 1$. As for the $\{1: 1 \rightarrow 2: 1\}$ flip, careful selection of the coupling interval is crucial to obtain the $\{2: 1 \rightarrow 1: 1\}$ flip in both model and experiment.

\section{E. Pause-induced $\{1: 1 \rightarrow 2: 1\}$ flip}

Mines showed a long time ago that a pause in stimulation produced by dropping two or three stimuli from the periodic drive train could result in the $\{1: 1 \rightarrow 2: 1\}$ flip in frog ventricle. ${ }^{12}$ We were able to reproduce this effect in only one of many cells in which a search for it was made. A typical unsuccessful trial is shown in Fig. 6, in which one [Fig. 6(A)] or two [Fig. 6(B)] stimuli were dropped. A 2:1 rhythm was not seen after the pause; instead there was transient alternans, followed by asymptotic re-establishment of 1:1 rhythm. The flip was, however, seen in one cell in which six stimuli were dropped [Fig. 6(C)]: a single transient 3:2 Wenckebach cycle was seen, followed by a maintained 2:1 rhythm. The action potential duration (APD) of the second action potential following resumption of stimulation was $\sim 10$ ms longer than during the pre-existing 1:1 rhythm, so that the third post-pause stimulus fell within the refractory 


\section{Experiment}
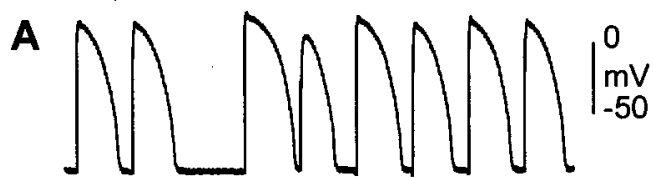

B
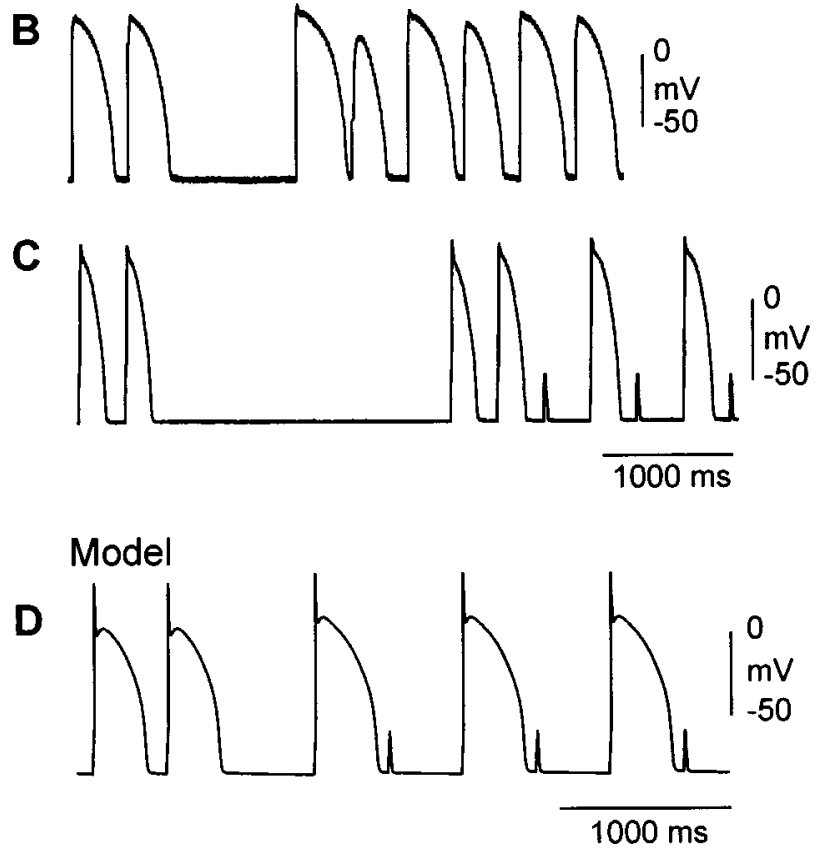

FIG. 6. Pause-induced $\{1: 1 \rightarrow 2: 1\}$ flip (experiment and model). (A)-(C): Experiment: One (A) or two (B) stimuli dropped from the basic drive train. $\mathrm{BCL}=430 \mathrm{~ms}$, pulse amplitude $=850 \mathrm{pA}$, pulse duration $=5 \mathrm{~ms}$. Same cell as in Figs. 1, 3(A), and 5(A). (C): Six stimuli dropped. Different cell from (A) and (B). $\mathrm{BCL}=345 \mathrm{~ms}$, pulse amplitude $=1000 \mathrm{pA}$, pulse duration $=10$ ms. Time-calibration bar in (C) applicable to (A) and (B). (D) Model: BCL $=430 \mathrm{~ms}$, pulse amplitude $=225 \mathrm{pA}$, pulse duration $=10 \mathrm{~ms}$. One stimulus dropped.

period of the second beat, resulting in a dropped beat and a flip to 2:1 rhythm. Dropping only one stimulus in this cell did not result in the $\{1: 1 \rightarrow 2: 1\}$ flip. In contrast, in the model it is necessary to omit only one stimulus pulse from the periodic drive train to obtain the $\{1: 1 \rightarrow 2: 1\}$ flip [Fig. 6(D)]. We believe that the ability to obtain the flip in the experiment depends on the presence of the transient outward current $\left(I_{\text {to }}\right)$ in our rabbit cells (see Discussion).

\section{F. Action potential duration recovery curve}

The existence of hysteresis, as well as the pulse- and pause-induced flips, has previously been attributed to the dependence of the action potential duration and refractory period on the prior history of stimulation. ${ }^{12}$ One way of characterizing the well-known dependence of APD and refractory period on BCL is to plot APD vs recovery time or diastolic interval at each BCL. ${ }^{16,35}$ The action potential duration (APD) was measured from the start of the injected stimulus pulse to the point in time on the repolarizing limb of the action potential at which the transmembrane voltage crossed $-60 \mathrm{mV}$. The diastolic interval or recovery time (RT) associated with a particular action potential was defined as the time interval between the point in time at which the
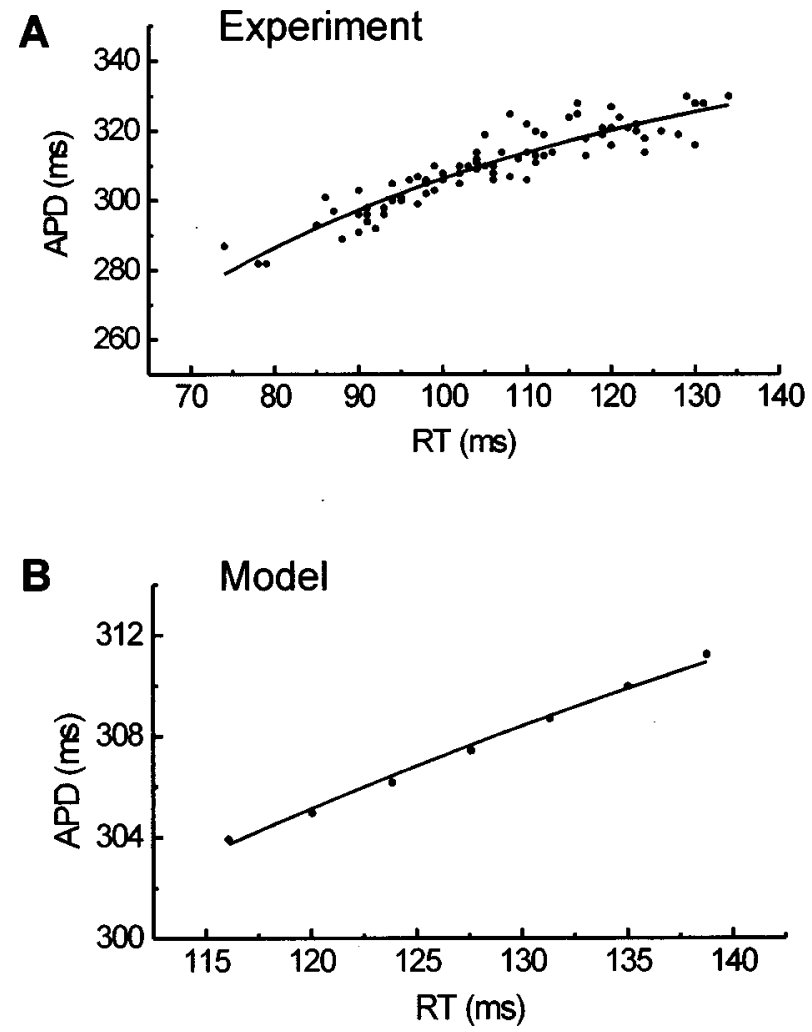

FIG. 7. Steady-state action potential duration recovery curve (APDRC) from 1:1 rhythm (experiment and model). (A) Experiment [data extracted from runs in Fig. 1(C)]. (B) Model [data extracted from runs in Fig. 2(C)]. APD measured from start of stimulus pulse to time at which $-60 \mathrm{mV}$ crossed on repolarizing limb of action potential. Recovery time measured from $-60 \mathrm{mV}$ on repolarizing limb of preceding action potential to start of stimulus pulse. Curves are single-exponential fits to the data points.

voltage on the repolarizing limb of the preceding action potential passed through $-60 \mathrm{mV}$ and the time at which the next stimulus pulse started. Figure 7(A) shows a plot of APD vs RT determined from the experiment of Fig. 1, with APD and RT determined during 1:1 rhythm for 10 action potentials at each of the eight BCLs in the range 380-450 ms (filled circles). The data was well fit by a single exponential curve

$$
\mathrm{APD}=g(\mathrm{RT})=A-B e^{-\left(\mathrm{RT}_{-}-\mathrm{RT}_{\text {min }}\right) / \tau}, \quad \mathrm{RT} \geqslant \mathrm{RT}_{\text {min }},
$$

where $A=352.42014 \mathrm{~ms}, B=73.35624 \mathrm{~ms}, \tau=55.73027$ $\mathrm{ms}$, and $\mathrm{RT}_{\min }=74.0 \mathrm{~ms} .^{18,36-38}$ The parameter $A$ can be interpreted as the asymptotic value of APD (i.e., APD in the limit $\mathrm{RT} \rightarrow \infty), \tau$ as the time constant of recovery of APD, and $\mathrm{RT}_{\text {min }}$ as the shortest $\mathrm{RT}$ possible at the smallest $\mathrm{BCL}$ for which there is 1:1 rhythm. The parameter $\mathrm{B}$ gives the range of APD obtainable. We shall refer to the curve in Fig. $7(\mathrm{~A})$ as the steady-state action potential duration recovery curve (APDRC). We also determined the APDRC in the LR model from the runs used to construct the hysteresis loop of Fig. 2(C) [Fig. 7(B)]. The APDRC was well fit by Eq. (1), with $A=366.12764 \mathrm{~ms}, B=62.09352 \mathrm{~ms}, \tau=174.74028$ $\mathrm{ms}$, and $\mathrm{RT}_{\min }=116.067 \mathrm{~ms}$ in the model [Fig. 7(B)]. 


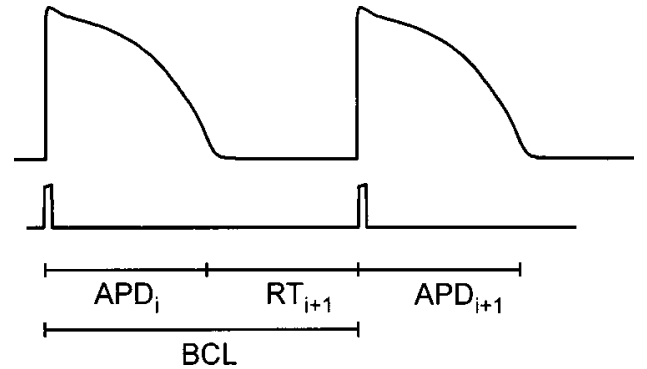

FIG. 8. Derivation of one-dimensional map. Diagram used in derivation of one-dimensional map: see text for further description.

\section{G. Derivation of a one-dimensional map from the recovery curve}

We shall now show how, under certain assumptions, the APDRC obtained during 1:1 rhythm can be used to predict the response to periodic stimulation at any arbitrary BCL. This involves deriving a one-dimensional finite-difference equation or "map" and carrying out iterations of that map. $^{35,39}$

Denote the APD of the $i$ th action potential obtained during periodic stimulation by $\mathrm{APD}_{i}$ and its associated recovery time by $\mathrm{RT}_{i}$. Let us first assume that during periodic stimulation at an arbitrary BCL, the duration of any action potential is determined only by the preceding RT, in a manner that is governed by the function $g$ in Eq. (1). One can then write

$$
\mathrm{APD}_{i+1}=g\left(\mathrm{RT}_{i+1}\right) \text {. }
$$

However, during periodic stimulation, one has, by definition of $\mathrm{APD}_{i}$ and $\mathrm{RT}_{i+1}$ (Fig. 8), that $\mathrm{BCL}=\mathrm{APD}_{i}+\mathrm{RT}_{i+1}$ and so

$$
\mathrm{RT}_{i+1}=\mathrm{BCL}-\mathrm{APD}_{i} .
$$

Substituting Eq. (3) into Eq. (2), one has

$$
\mathrm{APD}_{i+1}=g\left(\mathrm{RT}_{i+1}\right)=g\left(\mathrm{BCL}-\mathrm{APD}_{i}\right) .
$$

Equations (3) and (4) are true provided that there are no skipped beats, i.e., $\mathrm{RT}_{i+1} \geqslant \mathrm{RT}_{\min }$, the minimum possible $\mathrm{RT}$, or equivalently, from Eq. (3), that $\mathrm{APD}_{i} \leqslant \mathrm{BCL}$ $-\mathrm{RT}_{\min }$.

Let us now consider what happens when a skipped beat occurs. We now need to make our second assumption: in the event of a skipped beat the subthreshold membrane response does not affect the duration of the following action potential. If the $(i+1)^{s t}$ action potential is thus preceded by a skipped beat, we can therefore replace Eq. (3) with

$$
\mathrm{RT}_{i+1}=2 \mathrm{BCL}-\mathrm{APD}_{i}
$$

and Eq. (4) with

$$
\mathrm{APD}_{i+1}=g\left(\mathrm{RT}_{i+1}\right)=g\left(2 \mathrm{BCL}-\mathrm{APD}_{i}\right),
$$

for $\mathrm{BCL}-\mathrm{RT}_{\min }<\mathrm{APD}_{i} \leqslant 2 \mathrm{BCL}-\mathrm{RT}_{\min }$.

The general equation is thus

$$
\mathrm{APD}_{i+1}=g\left(\mathrm{RT}_{i+1}\right)=g\left(m(\mathrm{BCL})-\mathrm{APD}_{i}\right)=f\left(\mathrm{APD}_{i}\right),
$$

with $(m-1)$ being the number of skipped beats that occurred in $\mathrm{RT}_{i+1}$. This equation is a one-dimensional finite-
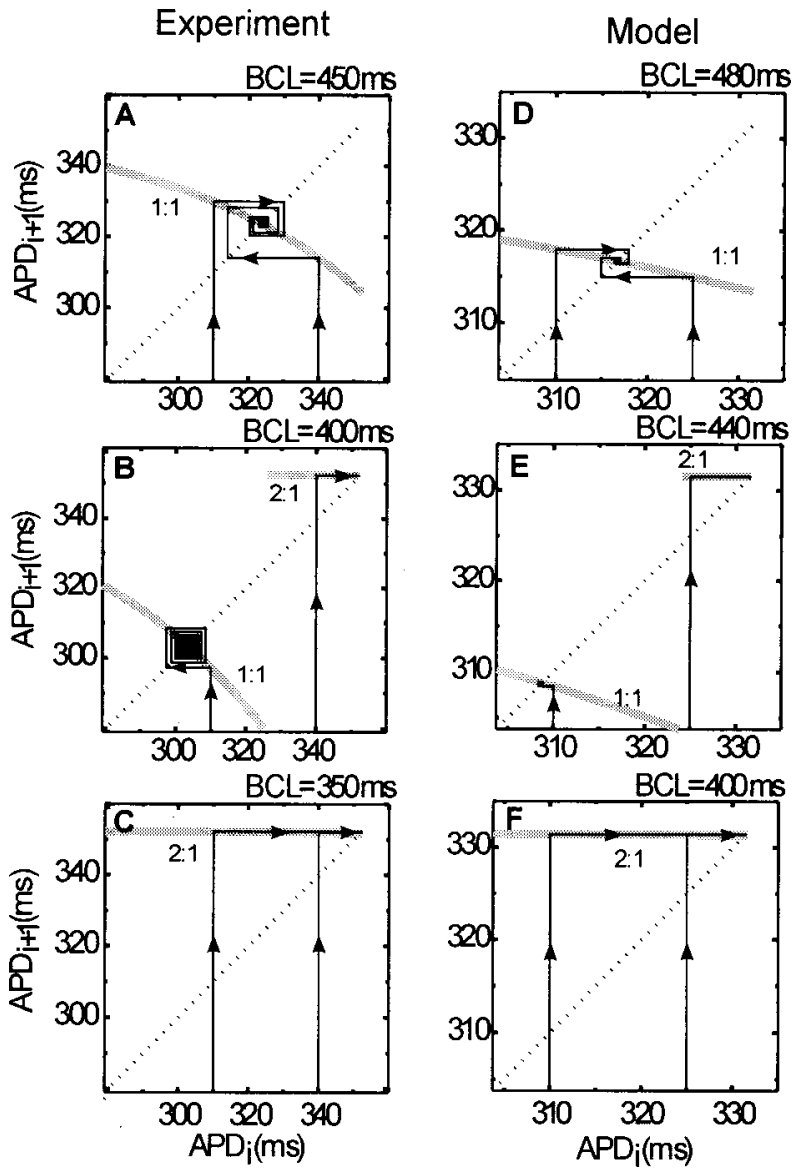

FIG. 9. Iteration of maps derived from the recovery curve (experiment and model). (A) $-(\mathrm{C})$ : Experiment. (A): $\mathrm{BCL}=450 \mathrm{~ms}$ : Iteration leads to a $1: 1$ rhythm, independent of initial condition $\left(\mathrm{APD}_{1}=310 \mathrm{~ms}, 340 \mathrm{~ms}\right.$ illustrated). (B): $B C L=400 \mathrm{~ms}$ : Iteration leads to either a $1: 1$ or $2: 1$ rhythm depending on choice of initial condition (e.g., $\mathrm{APD}_{1}=310 \mathrm{~ms}, 340 \mathrm{~ms}$, respectively). $(\mathrm{C}): \mathrm{BCL}=350 \mathrm{~ms}$ : Iteration leads to a 2:1 rhythm, independent of initial condition ( $\mathrm{APD}_{1}=310 \mathrm{~ms}, 340 \mathrm{~ms}$ illustrated). Range of APD attainable at this BCL is from $\sim 279.1 \mathrm{~ms}$ to $\sim 352.4 \mathrm{~ms}$ in (A) $-(\mathrm{C})$. (D)(F): Model. (D): $B C L=480$ ms: 1:1 rhythm. (E): $B C L=440 \mathrm{~ms}: 1: 1$ and 2:1 rhythms. (F): $B C L=400 \mathrm{~ms}: 2: 1$ rhythm. Range of APD is from $\sim 303.7 \mathrm{~ms}$ to $\sim 331.6 \mathrm{~ms}$.

difference equation or map, since $\mathrm{APD}_{i+1}$ is a function of $\mathrm{APD}_{i}$ alone. At a specific BCL, Eq. (7) can be iterated to generate the dynamics, starting out from any initial condition $\mathrm{APD}_{1}$, since the function $g$ is known from Eq. (1).

\section{H. Iterations of the map}

Figures $9(\mathrm{~A})-9(\mathrm{C})$ shows maps derived from the APDRC obtained in experiment [Fig. 7(A)]. At a very long BCL [Fig. 9(A)] the map (stippled curve) has only one branch (the 1:1 branch), given by Eq. (4). Starting out at an initial condition $\left(\mathrm{APD}_{1}\right)$ of $310 \mathrm{~ms}$, iterates asymptotically approach the stable steady state (or fixed point or equilibrium point) at $\mathrm{APD}_{i+1}=\mathrm{APD}_{i} \cong 324 \mathrm{~ms}$, corresponding to $1: 1$ rhythm (the condition for local stability of a steady state is that the slope of the map at the steady state be less than one in absolute value). This steady state is also globally attracting, since iterates from all initial conditions asymptotically approach this steady state [e.g., $\mathrm{APD}_{1}=340 \mathrm{~ms}$ is also shown in Fig. 9(A)]. Because of the negative slope of the 
map at the fixed point, the iterates approach this point in an alternating fashion, i.e., successive iterates fall on opposite sides of the fixed point (see also Fig. 4 in Refs. 16 and 39). This fact accounts for the transient alternans following the pause in Figs. 6(A) and 6(B), following the flip to 1:1 rhythm in Fig. 5(A), and immediately preceding the establishment of 1:1 rhythm in Fig. 1(B) (see also Fig. 18 of Ref. 12).

As BCL is lowered, the map becomes discontinuous, with a second branch (the 2:1 branch), given by Eq. (6), appearing to the right of the $1: 1$ branch [Fig. 9(B)]. This discontinuity at $\mathrm{APD}_{i}=\mathrm{BCL}-\mathrm{RT}_{\text {min }}$ occurs because $\mathrm{BCL}$ is now small enough so that a sufficiently long $\mathrm{APD}_{i}$ is now followed by an $\mathrm{RT}_{i+1}$ that is less than $\mathrm{RT}_{\text {min }}$, so that a skipped beat can occur at this BCL. Thus, for $\mathrm{APD}_{1}$ $>\mathrm{BCL}^{-\mathrm{RT}_{\text {min }}}$ [e.g., $\mathrm{APD}_{1}=340 \mathrm{~ms}$ in Fig. 9(B)], all iterates fall on the 2:1 branch, asymptotically approaching the second stable steady state at $\mathrm{APD} \cong 352 \mathrm{~ms}$, corresponding to 2:1 rhythm. Since the 2:1 branch also has a negative slope, the approach to this steady state is again alternating [not visible on scale of Fig. 9(B)]. For $\mathrm{APD}_{1} \leqslant \mathrm{BCL}-\mathrm{RT}_{\min }$ [e.g., $\mathrm{APD}_{1}=310 \mathrm{~ms}$ in Fig. 9(B)], successive iterates fall on the $1: 1$ branch, and the steady state at $\mathrm{APD} \cong 303 \mathrm{~ms}$, corresponding to $1: 1$ rhythm, is asymptotically approached in an alternating fashion. ${ }^{16,39}$ There is thus now the co-existence of two stable steady states (bistability), one corresponding to $1: 1$ rhythm and the other to $2: 1$ rhythm, each with its own basin of attraction $\left(\mathrm{APD}_{1} \leqslant \mathrm{BCL}-\mathrm{RT}_{\min }\right.$ and $\mathrm{APD}_{1}$ $>\mathrm{BCL}-\mathrm{RT}_{\min }$, respectively). As BCL is decreased further, the point of discontinuity in the map moves to the left, so that eventually the 1:1 branch, and thus its steady state, are no longer present [Fig. 9(C)]. The period-1 fixed point on the $2: 1$ branch, corresponding to $2: 1$ rhythm, is now globally attracting.

Figures 9(D)-9(F) shows that the maps derived from the APDRC of the model have dynamics that are qualitatively similar to that of the maps derived from experiment.

\section{Bifurcation diagrams}

Figure 9 showed individual examples of maps selected to illustrate the evolution in the qualitative features of the map as the BCL was changed. These examples were taken from calculations in which the BCL was systematically changed over a wide range with a $1 \mathrm{~ms}$ increment in BCL. In the search for bistability, iterations were carried out from 100 evenly spaced initial conditions at each BCL. The first 1000 iterates were discarded in order to allow transients to pass. Plotting the predicted steady-state APD as a function of BCL yields a bifurcation diagram (gray circles in Fig. 10). Superimposed on these predictions are the actual results of periodic stimulation runs (solid circles). In the case of 1:1 rhythm, the data from the periodic stimulation runs are very close to the predictions of the map derived from the APDRC. However, this agreement must exist, since the predictions were generated using the single exponential fit to data from periodic stimulation runs resulting in 1:1 rhythm. While the iterative technique predicts the presence of bistability in both experiment [Fig. 10(A)] and model [Fig. 10(B)], the pre-
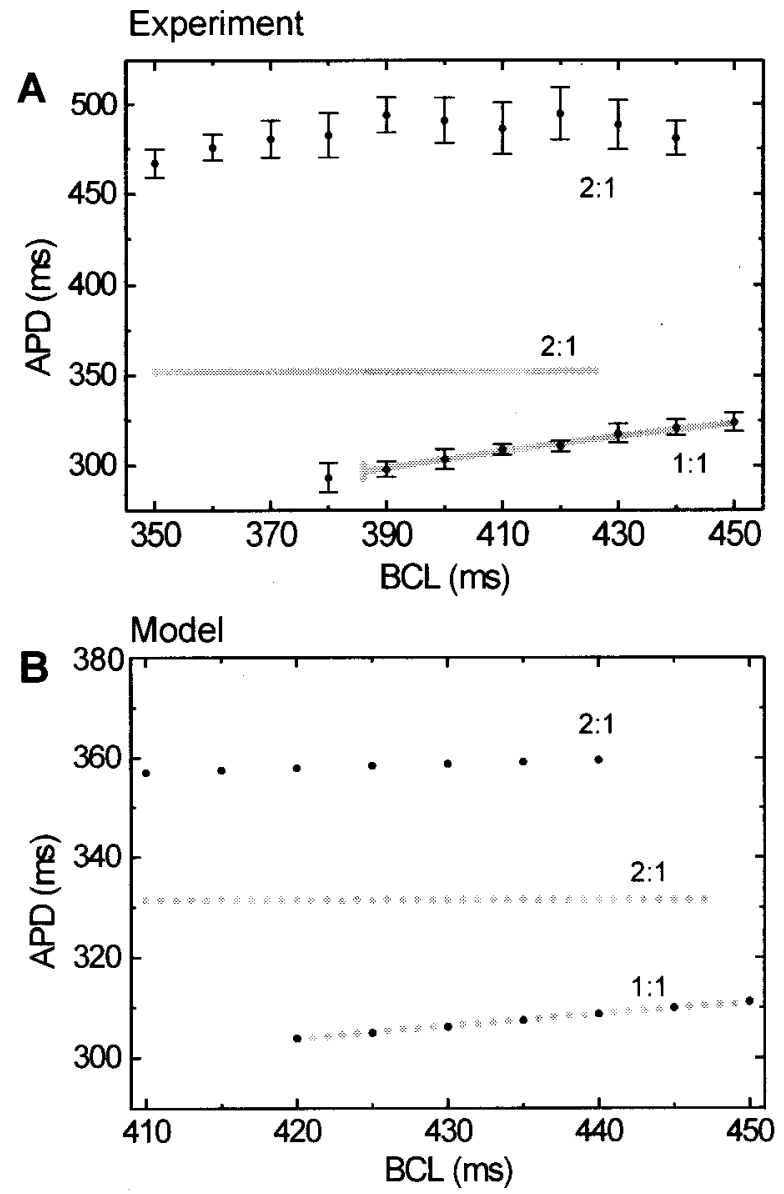

FIG. 10. Bifurcation diagrams obtained from iterations of map derived from recovery curves (experiment and model). (A): Experiment. (B): Model. Gray circles: Predicted APD from iterations carried out from 100 evenly spaced values of $\mathrm{APD}_{1}$ at each BCL. First 1000 iterates discarded to allow transients due to initial conditions to pass. Increment in $\mathrm{BCL}=1.0 \mathrm{~ms}$. Solid circles: Data taken from periodic stimulation runs of Figs. 1(C) and 2(C), with BCL decreasing for $1: 1$ points, increasing for $2: 1$ points. In (A), solid circles are mean APD of ten action potentials at each BCL; bars are \pm 1 standard deviation. At the shortest BCL on the 1:1 branch in (A), iterates from some initial conditions have not yet approached the steady state: however, they do so when iteration is continued beyond 1000 iterations.

dicted APD (gray circles) during 2:1 rhythm is considerably lower than the actual APD (solid circles) in both model and experiment.

This discrepancy in the model is due to the fact that the fit of the APDRC was made using data with RT $<140 \mathrm{~ms}$ [Fig. 7(B)], and this fit [dashed line in Fig. 11(A)] sharply underestimates the APD at the much longer RTs encountered during 2:1 rhythm [solid curve in Fig 11(A) shows fit to data (filled circles) computed during 1:1 rhythm over a much wider range of BCL: $420-1000 \mathrm{~ms}$ in Fig. 11(A) vs 420$450 \mathrm{~ms}$ in Fig. 7(B)]. Thus, the predicted APD during 2:1 rhythm in Fig. 10B is substantially less than the observed APD. Iterations using the APDRC fit to the data obtained over the wider range of BCL [solid curve in Fig. 11(A)] produce much better agreement for the APD during 2:1 rhythm [Fig. 11(B)]. The fact that the predicted APD is so close to the actual APD during 2:1 rhythm demonstrates that the two fundamental assumptions underlying the formulation 

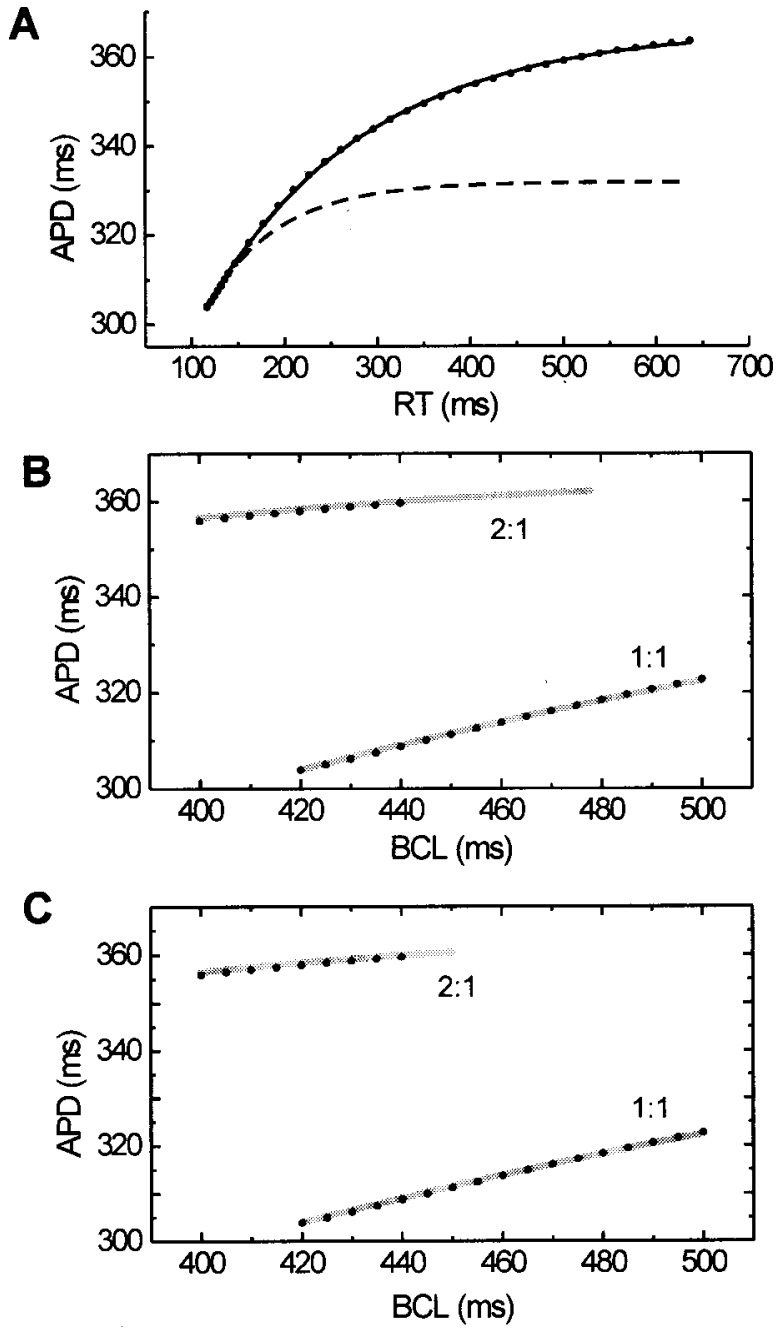

FIG. 11. APDRC determined over wider range of BCL and bifurcation diagram (model). (A): Steady-state APD vs RT data determined from runs at $\mathrm{BCL}=420-1000 \mathrm{~ms}$ (filled circles). Solid curve is a single-exponential fit to the data $[A=366.12764 \mathrm{~ms}, B=62.09352 \mathrm{~ms}, \tau=174.74028 \mathrm{~ms}$, and $\mathrm{RT}_{\min }=116.067 \mathrm{~ms}$ in Eq. (1)]. Dashed line indicates fit to restricted data set of Fig. 7(B) (BCL=420-450 ms). (B): Bifurcation diagram computed using APDRC of solid curve in (A). Gray symbols: Iterations carried out from 100 evenly spaced values of $\mathrm{APD}_{1}$ at each BCL. First 1000 iterates discarded to allow transients due to initial conditions to pass. Increment in $\mathrm{BCL}=1.0 \mathrm{~ms}$. Filled symbols: Data taken from periodic stimulation runs. (C): Bifurcation diagram computed as in (B), except that $\mathrm{RT}_{\min }=90.1 \mathrm{~ms}$ is used in computing the 2:1 branch with the iterative technique [the old value of $\mathrm{RT}_{\text {min }}$ $(116.067 \mathrm{~ms})$ is still used in the exponent in Eq. (1) to preserve the same dependence of APD on RT].

of the map are essentially correct in the LR model over the range of BCL investigated here: (i) $\mathrm{APD}_{i}$ is controlled by $\mathrm{RT}_{i}$ alone: i.e., little or no APD "memory,",18,36,40,41 which is also the case for closely related ventricular models; ${ }^{42,43}$ (ii) the subthreshold deflection caused by a stimulus that does not produce an action potential (a "blocked" stimulus) has no effect on the APD of the subsequent action potential.

The one discrepancy now remaining in Fig. 11(B) is that the upper limit of BCL to which 2:1 rhythm is predicted to occur (gray symbols) in the ionic model is significantly higher than that at which it actually occurs in the periodic stimulation runs (solid symbols). This leads to the predicted hysteresis range being significantly wider than the observed range ( $\sim 60 \mathrm{~ms}$ vs $\sim 20 \mathrm{~ms})$. This discrepancy is due to the fact that although $\mathrm{RT}_{\min }$ was assumed to be a constant in our iterative scheme, it is actually a function of BCL. While $\mathrm{RT}_{\text {min }}$ was defined as the RT encountered during steady-state 1:1 rhythm at the shortest BCL allowing 1:1 rhythm [Figs. 7(B) and 11(A)], an S1-S2 premature stimulation protocol revealed that the refractory period and the minimum possible RT were functions of the S1-S1 interval during 1:1 rhythm: as the S1-S1 interval increased, the APD and refractory period of the basic beat increased while the minimum possible RT decreased. In particular, a premature stimulation protocol carried out during 1:1 rhythm at an S1-S1 interval of $880 \mathrm{~ms}$ (corresponding to the effective cycle length prevailing in the middle of the predicted range of 2:1 rhythm in Fig. 11(B): $2 \times 440 \mathrm{~ms}$ ) demonstrated that the minimum RT was in fact only $90.1 \mathrm{~ms}$ at that $\mathrm{S} 1-\mathrm{S} 1$ interval. It is thus not surprising that the iterations in Fig. 11(B) predicted that the 2:1 rhythm should be maintained out to a longer BCL than in the simulations, since these calculations assumed a significantly higher value of $\mathrm{RT}_{\min }(\sim 116.1 \mathrm{~ms})$. When $\mathrm{RT}_{\min }$ was set to $90.1 \mathrm{~ms}$ and the iterations redone for the $2: 1 \mathrm{branch}$ of the bifurcation diagram, the upper border of the $2: 1$ zone was predicted to be at $\mathrm{BCL}=450 \mathrm{~ms}[\mathrm{Fig}$. 11(C)]. The iterative technique thus worked extremely well in the ionic model, predicting the APD almost perfectly within the 2:1 zone, and the location of the upper end of that zone to within 5-10 ms.

However, when $\mathrm{RT}_{\min }=90.1 \mathrm{~ms}$ was used in the calculation of the 1:1 zone, the left-hand border of that zone was at $\mathrm{BCL}=385 \mathrm{~ms}$, since $\mathrm{RT}_{\text {min }}$ was then unrealistically short for the briefer APD encountered at the lower BCL. Premature stimulation protocols showed that not only did the minimum RT depend on the BCL, but so did the detailed shape of the APD restitution curve. Thus, future extensions of the iterative technique should incorporate a parametric fit of the cycle-length-dependence of $\mathrm{RT}_{\text {min }}$ and the other parameters in Eq. (1) describing the APD restitution curve. The unfortunate cost of such an endeavor might be the loss of the nice one-dimensional nature of the map, with its easily visualized dynamics (Fig. 9).

The discrepancy between the iterative predictions and the actual APD during 2:1 rhythm is much larger in experiment than in the model (Fig. 10), with the predictions overestimating the measured APD by $\sim 115-145 \mathrm{~ms}$. This disparity is largely due to the fact that there is indeed an effect of the blocked stimulus during the experiments that is neglected in the iterative scheme. At any BCL within the 2:1 range of BCL shown in Fig. 10(A) (350-440 ms), the blocked stimulus falls on the repolarizing limb of the action potential, prolonging the duration of that action potential [see, e.g., first part of trace of Fig. 5(A)]. During the transient that occurs immediately following the conversion of $1: 1$ to 2:1 rhythm, the blocked stimulus falls after the action potential, and thus no prolongation effect is seen [e.g., Figs. 1(A) and $3(\mathrm{~A})]$. As stimulation proceeds, however, the APD gradually prolongs until the blocked stimulus falls on the action potential, as in Fig. 5(A). This prolongation effect does not occur in the model over the range of BCL investigated above (it does, however, occur at a higher pulse amplitude). 
For an action potential in which the prolongation effect occurs, let us denote the extrapolated intrinsic APD, neglecting the prolongation effect, by $\mathrm{APD}^{\prime}$, so that $\mathrm{APD}=\mathrm{APD}^{\prime}+\mathrm{P}$, where $\mathrm{P}$ is the prolongation caused by the blocked stimulus. Let us now assume that the effect of the blocked stimulus is simply to delay or postpone recovery from refractoriness, so that the start of the recovery time (RT) can be taken, as before, from the time at which the voltage crosses through $-60 \mathrm{mV}$ on the repolarizing limb of the action potential ("reset" effect). ${ }^{43}$ Let us then assume as before that the intrinsic APD is controlled by the preceding RT, so that $\mathrm{APD}_{i+1}^{\prime}=g\left(\mathrm{RT}_{i+1}\right)=g\left(2 \mathrm{BCL}-\mathrm{APD}_{i}\right)$. Substituting $\mathrm{APD}_{i+1}^{\prime}=\mathrm{APD}_{i+1}-P$, one then has $\mathrm{APD}_{i+1}$ $=g\left(2 \mathrm{BCL}-\mathrm{APD}_{i}\right)+\mathrm{P}$. Since $\mathrm{P}$ is on the order of several tens of milliseconds in our experiments, the prolongation effect accounts for only a part of the discrepancy (115-145 ms) between the predicted and actual APD in Fig. 10(A).

It is possible that the residual discrepancy is due to the same two factors producing the discrepancy in the model (i.e., APDRC determined over too narrow a range of BCL, thus underestimating the APD at the longer RTs encountered during 2:1 rhythm [as in Fig. 11(A)]; $\mathrm{RT}_{\text {min }}$ dependent on $\mathrm{BCL}$ ). However, since our stimulation protocol in the experiments was not set up to systematically explore longer BCL's, nor to determine the nature of the dependence of the minimum RT on BCL during 1:1 rhythm, we are unable to verify that this is also the case in the experiments. Indeed, it is our impression that carrying out such a definitive systematic protocol would take so long that serious concerns about the stationarity of the single-cell preparation in the classic whole-cell recording mode would be raised, due to dialysis of intracellular contents against the pipette fluid and continual rundown of currents. ${ }^{34}$ However, three bits of evidence suggest that the resolution of the discrepancy in the experiments might be similar to that given above in the case of the ionic model. First, we know that the fitted experimental APDRC [curve in Fig. 7(A)] underestimates the actual APD during 1:1 rhythm at $\mathrm{BCL}=1000 \mathrm{~ms}$, since we have data in some cells at this one long BCL (the stimulus threshold for 1:1 rhythm reported on earlier was obtained at this BCL). Second, the fit also sharply underestimates $\mathrm{APD}^{\prime}$ at the much longer RTs encountered during 2:1 rhythm: for the 2:1

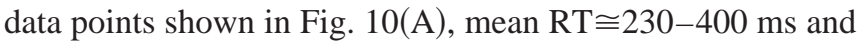
mean $\mathrm{APD}^{\prime} \cong 420-440 \mathrm{~ms}$; in contrast the fit predicts $\mathrm{APD}^{\prime} \cong 350 \mathrm{~ms}$ over this entire range of recovery time. Third, it is well known that the refractory period is a function of BCL in multicellular ventricular muscle. ${ }^{18}$

\section{J. Amplitude hysteresis}

While the $\{1: 1 \leftrightarrow 2: 1\}$ transitions seen in Figs. 1 and 2 above were produced by changing the BCL or equivalently the frequency of stimulation ("frequency hysteresis"), ${ }^{27}$ hysteresis could also be produced by changing the stimulus amplitude at a fixed BCL ("amplitude hysteresis"). ${ }^{27} \mathrm{~A}$ direct $\{1: 1 \rightarrow 2: 1\}$ transition [Fig. 12(A), upper panel] and a direct $\{2: 1 \rightarrow 1: 1\}$ transition [Fig. 12(B), lower panel] occurred with a change in stimulus amplitude when the BCL was set appropriately (arrows indicate first stimulus at new
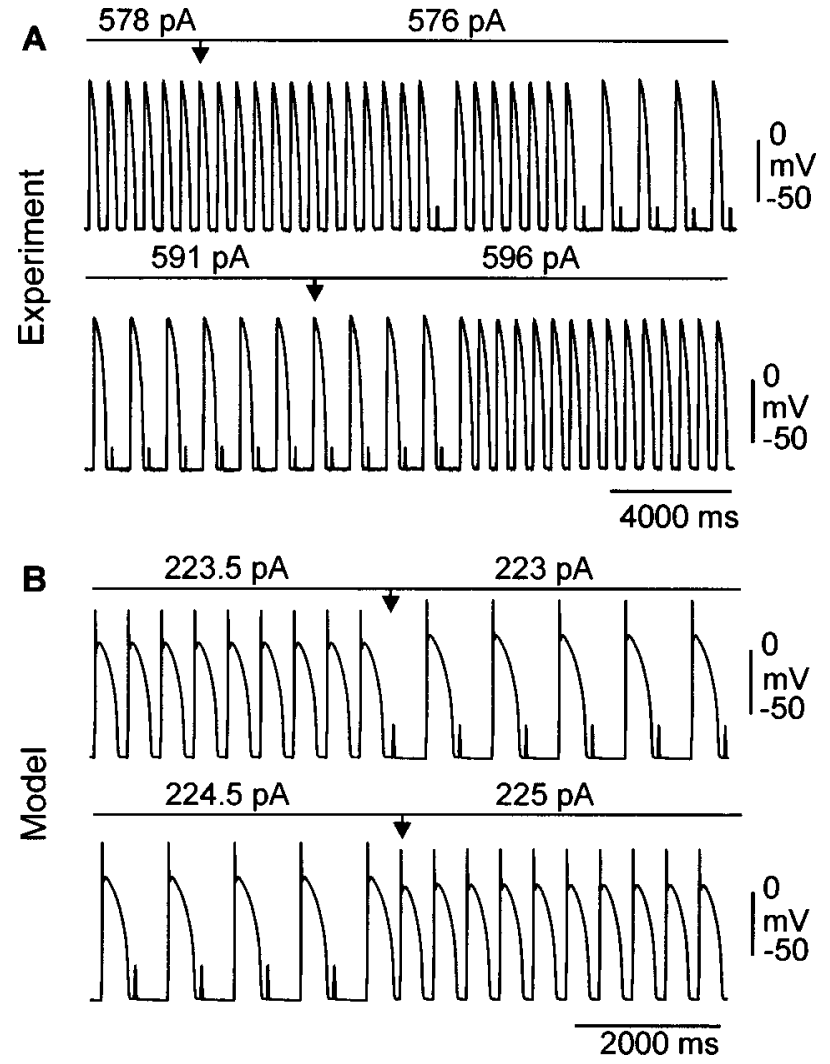

FIG. 12. Amplitude hysteresis (experiment and model). (A) Experiment. Upper trace: $\{1: 1 \rightarrow 2: 1\}$ transition when stimulus amplitude lowered from 578 to 576 pA. Lower trace: $\{2: 1 \rightarrow 1: 1\}$ transition when stimulus amplitude was later increased from 591 to $596 \mathrm{pA}$. BCL $=600 \mathrm{~ms}$, stimulus duration $=10 \mathrm{~ms}$. (B): Model. Upper trace: $\{1: 1 \rightarrow 2: 1\}$ transition when stimulus amplitude lowered from 223.5 to 223.0 pA. Lower trace: $\{2: 1 \rightarrow 1: 1\}$ transition when stimulus amplitude later increased from 224.5 to $225.0 \mathrm{pA}$. BCL $=450 \mathrm{~ms}$, stimulus duration $=10 \mathrm{~ms}$. From our standard initial conditions (see Methods) the stimulus amplitude was increased from $225 \mathrm{pA}$ to $230 \mathrm{pA}$ for 20 stimuli, and then lowered down to $223 \mathrm{pA}$ in steps of $0.5 \mathrm{pA}$, injecting 20 stimuli at each amplitude; then it was increased up to $225 \mathrm{pA}$ in steps of $0.5 \mathrm{pA}$, injecting 20 stimuli at each amplitude. Arrow points to time at which pulse amplitude was changed.

pulse amplitude). Since the $\{2: 1 \rightarrow 1: 1\}$ transition occurred at a higher stimulus amplitude than the $\{1: 1 \rightarrow 2: 1\}$ transition, there was amplitude hysteresis. Similar behavior was also seen in the LR model [Fig. 12(B)]. As in the case of frequency hysteresis, there was a rather long delay between the change in stimulus amplitude and the switch from one rhythm to the other in the experiments [Fig. 12(A)], but not in the model [Fig. 12(B)]. Just as the stimulus amplitude must be well chosen to obtain $\{1: 1 \leftrightarrow 2: 1\}$ frequency hysteresis, the BCL must also be carefully selected to obtain $\{1: 1 \leftrightarrow 2: 1\}$ amplitude hysteresis: e.g., if the BCL is set to $1000 \mathrm{~ms}$, one typically obtains Wenckebach rhythms in our rabbit ventricular cells instead of the direct transition to $2: 1$ rhythm as pulse amplitude is decreased. ${ }^{26}$

We now outline the mechanisms we deduce underlying $\{1: 1 \leftrightarrow 2: 1\}$ amplitude hysteresis. During 2:1 rhythm at a given BCL, the APD is longer than during 1:1 rhythm established at that same BCL at a higher pulse amplitude (but lying within the amplitude hysteresis range). Therefore, during 2:1 rhythm, the recovery time from the end of an action potential to the beginning of the following subthreshold re- 
sponse is shorter than the corresponding RT during 1:1 rhythm. Since the stimulus producing the subthreshold response during 2:1 rhythm falls within the relative refractory period of the preceding action potential, increase of stimulus amplitude will eventually convert that response into an action potential, thereby producing 1:1 rhythm (provided that the BCL be appropriate to the direct $\{2: 1 \rightarrow 1: 1\}$ transition). However, due to the smaller recovery time prevailing during the initial 2:1 rhythm, in order to obtain the conversion to $1: 1$ rhythm, it is necessary to raise the pulse amplitude to a value higher than that needed to maintain a 1:1 rhythm already established at that BCL.

\section{DISCUSSION}

\section{A. The direct $\{1: 1 \rightarrow 2: 1\}$ transition}

There are several cardiac preparations in which the direct $\{1: 1 \rightarrow 2: 1\}$ transition has been documented in response to decreasing $\mathrm{BCL}^{12,13,25,32,33,44,45}$ or stimulus amplitude. ${ }^{44,46}$ In other experimental reports, the BCL or stimulus amplitude was not changed with a fine enough decrement (e.g., 50 or $100 \mathrm{~ms}$ decrement in BCL used in Ref. 14) to guarantee that the transition was direct (i.e., that no intermediary Wenckebach, alternans, or other rhythms existed). There have also been two reports of the direct $\{1: 1 \rightarrow 2: 1\}$ transition in ionic models: one in a modified version of the Beeler-Reuter model of space-clamped ventricular membrane, ${ }^{42}$ the other in a one-dimensional Purkinje fiber cable. ${ }^{47}$ In both of these studies, and in the one experimental study in which stimulus amplitude was systematically changed, ${ }^{33}$ the direct $\{1: 1 \rightarrow 2: 1\}$ transition was seen at a stimulus amplitude lower than that at which alternans rhythm was produced. In addition, Wenckebach rhythms were seen at a lower amplitude (very close to threshold) in ventricular ionic models, ${ }^{23,29}$ as well as in the Purkinje fiber cable model when the fast sodium current $\left(I_{\mathrm{Na}}\right)$ was removed and intercellular coupling decreased, which had the effect of reducing excitability and the effective amplitude of the stimulus current. ${ }^{47}$ We find that lowering the pulse amplitude in the modified LR model below that which we have used above leads to Wenckebachtype rhythms when BCL is decreased (see also Ref. 29), while raising it leads to a sustained alternans rhythm. Thus, all of the available experimental and modeling evidence points to the same conclusion: when BCL is changed, the direct $\{1: 1 \rightarrow 2: 1\}$ transition is seen only when the effective stimulation amplitude is at an intermediate level.

\section{B. $\{1: 1 \leftrightarrow 2: 1\}$ frequency hysteresis}

Frequency hysteresis involving 1:1 and 2:1 rhythms has been described previously in three different cardiac preparations: Frog ventricle, ${ }^{12,14}$ dog ventricle, ${ }^{13}$ and aggregates of embryonic chick ventricular cells. ${ }^{32,33}$ The first of these studies was carried out almost a century ago by Mines, who recorded contractions in frog ventricle. ${ }^{12}$ Mines' explanation for the hysteresis (given in Results above) lay in the dependence of the refractory period on BCL. Our recording of the transmembrane potential in isolated cells lends credence to these mechanisms suggested by Mines. We are not aware of any previous reports of $\{1: 1 \leftrightarrow 2: 1\}$ frequency hysteresis in an ionic model.

\section{C. $\{1: 1 \leftrightarrow 2: 1\}$ amplitude hysteresis}

We know of one prior report of $\{1: 1 \leftrightarrow 2: 1\}$ amplitude hysteresis in cardiac tissue, in experiments carried out on Purkinje fibre. ${ }^{46}$ Perhaps most interestingly, "negative", hysteresis, associated with "relative" supernormal excitability, could be seen in several preparations: i.e., the transition from 1:1 to $2: 1$ rhythm occurred at a higher stimulus amplitude than the reverse transition. A multivariable differencedifferential model using the recovery curves for APD and excitability (together with a slowly changing memory function for APD) could produce either positive or negative hysteresis, depending on parameter values. ${ }^{46}$ While the additional memory-dependent terms with long time constants are apparently necessary to generate the amplitude hysteresis described in this model, this is not the case for the frequency hysteresis seen by us. We are not aware of any prior reports of $\{1: 1 \leftrightarrow 2: 1\}$ amplitude hysteresis in an ionic model.

\section{The extrastimulus-induced $\{1: 1 \rightarrow 2: 1\}$ and $\{2: 1 \rightarrow 1: 1\}$ flips}

While there are experimental and clinical reports of extrastimulus-induced flips in experimental and clinical work, we are not aware of any such reports in an ionic model.

We know of only one report of an extrastimulus-induced $\{1: 1 \rightarrow 2: 1\}$ flip, in a patient with $2: 1$ atrioventricular heart block. $^{48}$ Injection of a sufficiently premature atrial or ventricular extrastimulus during sinus rhythm resulted in infraHisian block of that beat. Following the resultant post-extra systolic ventricular pause, the next sinus beat conducted, but the next one did not, thus setting up a rhythm of $2: 1$ block. The extrastimulus, therefore, induces a pause-dependent $\{1: 1 \rightarrow 2: 1\}$ flip essentially equivalent to that originally seen by Mines in frog ventricle ${ }^{12}$ and later on by us in the ionic model [Fig. 6(D)]. In the pulse-induced $\{1: 1 \rightarrow 2: 1\}$ flips of Figs. 3 and 4, it is not a post-extrasystolic pause that is involved, but rather a pause associated with a skipped beat caused by extrastimulus-induced prolongation of APD.

There are two reports of the $\{2: 1 \rightarrow 1: 1\}$ flip, one experimental $^{12}$ and one clinical. ${ }^{48}$ Our results in Fig. 5 are in agreement with the mechanisms recently deduced from Hisbundle recordings of the $\{2: 1 \rightarrow 1: 1\}$ flip: the postextrasystolic pause cannot be too long, otherwise the APD of the next action potential would be so long that the stimulus following that action potential would be blocked, thus reestablishing 2:1 block. ${ }^{48}$ While this reasoning implies that the APD of the extrasystole cannot be too short, we also wish to point out that it cannot be too long either, otherwise the stimulus following the extrasystole might block, reinstituting $2: 1$ block. The $\{2: 1 \rightarrow 1: 1\}$ flip shown in Fig. 5 is different from that of Mines, ${ }^{12}$ in that here the APD of the extrasystole is short enough so that the next beat is not blocked, as in Mines' scenario, resulting in a more immediate transition to 2:1 rhythm here than in Mines' case. Traces corresponding to Mines' case can, however, be seen in our single cells 
when a stimulus pulse is delivered later than in Fig. 5(A), so as to produce a longer action potential. However, the extrastimulus cannot be too late, otherwise the second stimulus following the extrastimulus also finds itself in the refractory period and the flip does not occur.

While we have not attempted to obtain flips within the hysteresis range produced by changing stimulus amplitude, we predict that they should exist: e.g., injecting a premature stimulus during 2:1 rhythm following a skipped beat should result in a flip to $1: 1$ rhythm in a manner similar to that shown in Fig. 5, provided that the APD of the premature action potential be sufficiently brief in duration, and dropping one or more stimuli during 1:1 rhythm might result in a flip to $2: 1$ rhythm.

\section{E. The pause-induced $\{1: 1 \rightarrow 2: 1\}$ flip}

We are aware of two experimental studies documenting a pause-induced $\{1: 1 \rightarrow 2: 1\}$ flip. ${ }^{12,33}$ For these results, and for our modeling result [Fig. 6(D)], Mines' initial explanation still holds: the first action potential following the pause in Fig. 6(D) has an APD and refractory period longer than those of action potentials during the pre-existing 1:1 rhythm; when the pause is sufficiently long, the refractory period associated with this beat will be so long that the second stimulus delivered following the pause will result in a blocked beat, producing a 2:1 cycle. The third stimulus will then be preceded by a recovery time long enough to allow it to elicit an action potential. The APD and refractory period of this beat will also be long, because of the relatively long recovery time, so that the fourth stimulus will result in a subthreshold response, and so forth, resulting in a sustained 2:1 rhythm.

In contrast to our modeling result [Fig. 6(D)] and to earlier experimental results in frog ventricle ${ }^{12}$ and embryonic ventricular cells, ${ }^{32,33}$ we have not been able to obtain the $\{1: 1 \rightarrow 2: 1\}$ flip by dropping one or two stimulus pulses in our experiments. The APD of the first beat following resumption of stimulation in the cell of Figs. 6(A) and 6(B) was only weakly dependent on the previous RT. The APD of this beat did not prolong enough to cause block of the next beat and thus induction of 2:1 rhythm [unlike the model, Fig. 6(D)]. However, following the very long recovery time produced by dropping several stimuli [Fig. 6(C)], the first post-pause beat had a more prominent phase- 1 early repolarization, and the APD of the second post-pause beat was about $10 \mathrm{~ms}$ longer than during 1:1 rhythm, so that its refractory period was extended and the third beat was blocked. When a single stimulus is dropped in rabbit ventricular cells during 1:1 rhythm at the longer BCL of $1000 \mathrm{~ms}$, there is a more prominent phase-1 repolarization of the first action potential and an increase in the duration of the second action potential (see Fig. 2 of Ref. 26). We have attributed these two changes to the presence of the transient outward current $\left(I_{\text {to }}\right)$, which has an extremely long time-constant $(\sim 7 \mathrm{~s})$ for recovery from inactivation in our cells. ${ }^{26}$ In fact, it is this current that is largely implicated in generating Wenckebach rhythms: the 3:2 cycle occurring right after the pause in Fig. 6(C) bears a striking resemblance to a cycle of maintained $3: 2$ Wenckebach rhythm of the kind we have reported at BCL
$=1000 \mathrm{~ms}^{26}$ The absence of $I_{\mathrm{to}}$ in the LR model means that the above effects will not be present. Due to its long time constant, $I_{\text {to }}$ is not likely to be a key player in our preparation during behavior occurring at short BCLs and recovery times (e.g., the hysteresis itself and the pulse-induced flips).

\section{F. Ionic mechanisms underlying $\{1: 1 \leftrightarrow 2: 1\}$ bistability}

Two basic electrophysiological properties underlie the $\{1: 1 \leftrightarrow 2: 1\}$ bistability in the model. First, at the stimulus amplitude used here, there is effectively an all-or-none threshold, which in a ventricular cell hinges on a competition between the depolarizing influence of the fast inward current $\left(I_{\mathrm{Na}}\right)$ and the repolarizing influence of the inwardly rectifying potassium current $\left(I_{\mathrm{K} 1}\right)^{49}$ (see Ref. 50 for a detailed investigation in the Hodgkin-Huxley model of squid axon). Second, as in the Beeler-Reuter model from which the LR model is derived, there is a time-dependent recovery of APD, which hinges on two time-dependent processes: activation of the delayed rectifier current $\left(I_{\mathrm{K}}\right)$ and recovery from inactivation of the slow inward calcium current $\left(I_{\mathrm{si}}\right) .^{51,52}$ Indeed, in isolated rabbit ventricular cells studied at room temperature in the whole-cell mode, the time constant for recovery of APD was very close to the time constant for recovery from inactivation of the calcium current. ${ }^{37}$ While our understanding of the ionic basis underlying the ventricular action potential and the restitution of APD is still very much incomplete, it is clear that mechanisms not expressly included in the LR model [e.g., $\mathrm{Na}-\mathrm{Ca}$ exchanger, ${ }^{38,53} \mathrm{Na}-\mathrm{K}$ pump, ${ }^{54}$ transient outward current $\left.\left(I_{\text {to }}\right)^{26}\right]$ also contribute to varying extents in different species. Future modeling work investigating the ionic basis of bistability and hysteresis should thus utilize a species-specific "second-generation' model incorporating the $\mathrm{Na}-\mathrm{Ca}$ exchanger, the $\mathrm{Na}-\mathrm{K}$ pump, and additional currents such as $I_{\text {to }}$. However, the problems of drift ${ }^{55}$ and degenerate equilibria ${ }^{56,57}$ now known to exist in the original formulation of this class of models will have to be addressed before this approach can be taken.

\section{G. $\{1: 1 \leftrightarrow 2: 1\}$ bistability on the map}

Our derivation of a one-dimensional map obtained from the APDRC provides a theoretical framework in which one can consider $\{1: 1 \leftrightarrow 2: 1\}$ bistability (see also Refs. 14 and 46 ). This approach allows a quantitative test of Mines' explanation that the essential mechanism underlying the $\{1: 1 \leftrightarrow 2: 1\}$ hysteresis, as well as the extrastimulus- and pause-induced flips, is the dependence of APD and refractory period on BCL. It also supports the interpretation that the $\{1: 1 \leftrightarrow 2: 1\}$ hysteresis is a consequence of the predicted coexistence of two stable equilibrium points on the map [Figs. 9(B) and $9(\mathrm{E})]$. In the succinct words of Mines: "It is seen that over a quite considerable range of frequencies of excitation, there exist two possible equilibria, stable so long as the heart continues beating regularly and without interruption.",12

The $\{1: 1 \leftrightarrow 2: 1\}$ bistability hinges on the APDRC having a nonzero, but not too large, slope. Should the APDRC be perfectly flat (i.e., no time-dependence of APD restitution), there would still be a direct $\{1: 1 \rightarrow 2: 1\}$ transition, but no bistability. As the APDRC is made increasingly curved, one 
would see $\{1: 1 \leftrightarrow 2: 1\}$ bistability and the range of BCL over which this bistability occurs would initially increase. In our simple iterative scheme, when the APDRC steepens to the point where there is a region of slope $>1$, there will be a period-doubling bifurcation, corresponding to a transition from $1: 1$ to $2: 2$ rhythm. ${ }^{14,16,20,35,42,58,59}$ However, a recent report shows the existence of the direct $\{1: 1 \rightarrow 2: 1\}$ transition in a two-dimensional map (incorporating APD memory as the second variable), even when the slope of the steady-state APDRC exceeds 1 over some range. ${ }^{14}$ Nevertheless, the $\{1: 1 \leftrightarrow 2: 1\}$ transition can be accounted for by a two-branched one-dimensional map. ${ }^{27,45,58}$

\section{H. Slow transients and memory}

A long transient is seen in our experiments between the time at which BCL is changed and the time at which a switch between 1:1 and 2:1 rhythms is observed (Figs. 1 and 12). There is also a transient immediately following the switch to $1: 1$ rhythm [Fig. $1(\mathrm{~A})]$ or immediately after the $\{2: 1 \rightarrow 1: 1\}$ flip [Fig. 3(A)], with the APD slowly prolonging to the point where the subthreshold deflection eventually rides on the action potential, as in Fig. 5(A). Such prolonged transients are not seen in the ionic model [Figs. 2(A), 2(B), 3(B), 5(B), and $6(\mathrm{D})]$ or in the one-dimensional map. These transients indicate the presence of processes with long time constants. It is thus possible that including effects extending over several beats that are not presently incorporated into the ionic model (e.g., pumps, exchangers, time-varying ionic concentrations) or into the simple one-dimensional map (e.g., memory of excitability and APD) ${ }^{14,36,40,41,45,46,60-62}$ might replicate such long transients. However, there are practical experimental difficulties (e.g., rundown of currents) inherent in sorting out these slow phenomena in the single-cell preparation.

\section{Shortcomings of the iterative technique}

As mentioned earlier (see Results), we are not sure of the reason(s) underlying the failure of the iterative technique to more accurately predict the APD during 2:1 rhythm [Fig. $10(\mathrm{~A})]$. One possibility not mentioned thus far is that incorporation of slow processes (e.g., APD memory) would help in resolving this discrepancy. However, a model incorporating APD memory [but not explicit BCL-dependence of $\mathrm{RT}_{\text {min }}$, as in Fig. 11(C)] also has significant discrepancies in its predictions (e.g., predicted APD too large [vs too small in Fig. 10(A)] during 2:1 rhythm; predicted upper end of 2:1 range at much too long a BCL). ${ }^{14}$ In that study on frog ventricle, blocked stimuli fall on the repolarizing limb of the action potential during 2:1 rhythm [Fig. 1(b) of Ref. 14]. Depolarizing pulses injected into ventricular muscle can result in either a prolongation or shortening of the APD, depending on the amplitude, duration, and timing of the stimulus, as well as the species. ${ }^{63}$ Should shortening of APD occur in frog ventricle, incorporation of such a "reset" effect into the iterative scheme might help in improving the predictions, as in our work above and in an ionic model. ${ }^{43,59}$

\section{J. Other forms of bistability in cardiac electrophysiology}

While we have concentrated above on $\{1: 1 \leftrightarrow 2: 1\}$ bistability, several other forms of bistability involving two different periodic rhythms have been described in experimental cardiac electrophysiology. In particular, there are several examples involving $1: 1,2: 2$, and $2: 1$ rhythms.

The transition $\{1: 1 \rightarrow 2: 2 \rightarrow 2: 1\}$ is seen when our cells are paced with a stimulus amplitude higher than that used above. In that situation, the $2: 1$ rhythm is bistable with either the $1: 1$ or the $2: 2$ rhythm, depending on the BCL, with both hysteresis and flips being seen. ${ }^{27}$ This form of hysteresis has also been more recently reported in frog ventricle. ${ }^{14}$ In a onedimensional cable model of ventricular muscle, either a 2:1 or 2:2 rhythm is seen, dependent on initial conditions, thus directly demonstrating bistability. ${ }^{58}$ Before it was described in experiments and ionic models, this form of bistability involving the $\{1: 1 \rightarrow 2: 2 \rightarrow 2: 1\}$ sequence of transitions was theoretically predicted to exist based on iterations of a onedimensional map (Ref. 35; see also Refs. 14, 58, and 59).

In frog ventricle, there was a transition from 1:1 to what was described as one of two different 2:2 rhythms (to our eye they look more like a $2: 2$ rhythm and either a $2: 1$ or $4: 2$ rhythm), depending on the exact point in time at which the BCL was lowered. ${ }^{16}$ Iterations of a one-dimensional map obtained from consideration of an ionic model also predicted the existence of two stable 2:2 rhythms [Figs. 5 and 6(d) of Ref. 59; see also Fig. 8(b) of Ref. 58]. An interesting form of multistability or neutral stability occurs in mechanical alternans: at a fixed BCL, the amplitude of the contraction can attain many different values, depending on the length of a single longer-than-average cycle that is inserted. ${ }^{39}$ Finally, Mines gives a very beautiful example in which a well-timed extrastimulus converts 2:1 bilateral bundle branch block into an alternating bundle branch block or vice versa. ${ }^{12}$

Another form of bistability is $\{1: 1 \leftrightarrow 1: 0\}$ bistability. When the denervated in situ canine heart is paced from the right ventricle, there is hysteresis in that 1:1 ventricular capture is attained and lost at different frequencies of stimulation. ${ }^{13}$ There is also amplitude hysteresis in the threshold for "capture", when an electronic pacemaker is inserted into a patient: i.e., the threshold current is slightly higher when stimulus amplitude is increased slowly from a subthreshold value than when it is decreased from a suprathreshold value. ${ }^{64}$ While it has been suggested that the decreased threshold found when slowly decreasing stimulus amplitude during 1:1 rhythm is due to the higher coronary blood flow then present, ${ }^{64}$ this $\{1: 1 \leftrightarrow 1: 0\}$ hysteresis can also be seen in isolated slow-response rabbit atrium (where it has been termed "excitation hysteresis'"), ${ }^{60}$ isolated papillary muscle, ${ }^{46}$ single guinea-pig ventricular cells, ${ }^{61}$ a modified version of the LR model, ${ }^{62}$ an "analytic'" model, ${ }^{61}$ and a differential-difference model. ${ }^{46}$ In sheep papillary muscle, ${ }^{65}$ as well as in the modified version of the LR model, ${ }^{62}$ injection of an additional stimulus (which is, however, subthreshold) during 1:1 rhythm can produce the $\{1: 1 \rightarrow 1: 0\}$ flip; an additional suprathreshold stimulus delivered during 1:0 rhythm can produce the $\{1: 0 \rightarrow 1: 1\}$ flip in the model. ${ }^{62}$ To obtain $\{1: 1 \leftrightarrow 1: 0\}$ bistability in the LR model, it is apparently 
essential to add a slow gating process to the inward rectifier current $\left(I_{\mathrm{K} 1}\right){ }^{62}$ In contrast, we have shown that it is not necessary to add any extra slow process to the original LR model to obtain $\{1: 1 \leftrightarrow 2: 1\}$ bistability.

\section{K. Implications for arrhythmias}

\section{Atrioventricular block}

Two types of second-degree atrioventricular (AV) block have been described. In Mobitz type I or Wenckebach block, there is a beat-to-beat increment of the PR interval preceding the skipped beat; in Mobitz type II block, there is an occasional dropping of a ventricular beat with no detectable trend in the PR intervals of the beats immediately preceding the dropped beat. A unified hypothesis for the two forms of Mobitz block has been proposed, with dropped beats in Mobitz type II block occurring within the proximal His-Purkinje system actually being preceded by very small increments in conduction time ("millisecond Wenckebach"). ${ }^{66}$ As the degree of block worsens, the increments in the PR interval increase to the point where frank Mobitz type I block is readily identified.

Our findings in single ventricular cells show several correspondences with AV block. First, we see Wenckebach rhythms only at a pulse amplitude lower than that at which the direct $\{1: 1 \rightarrow 2: 1\}$ transition is seen. Second, during the $\{1: 1 \rightarrow 2: 1\}$ transition produced by gradually lowering the stimulus amplitude at fixed BCL, there is a very slight increase in latency - which would probably translate into an increase in conduction time in a multicellular preparation preceding the transition to $2: 1$ rhythm [Fig. 12(A), upper panel]. Third, a slight lowering of the stimulus amplitude after the $\{1: 1 \rightarrow 2: 1\}$ transition has occurred following a decrease in stimulus amplitude results in a transition to 1:0 block, which agrees with the propensity for paroxysmal complete AV block to occur following Mobitz type II block in the His-Purkinje system. ${ }^{66,67}$ Finally, a very recent clinical report shows that a single extrastimulus can induce flips from 1:1 conduction to $2: 1 \mathrm{AV}$ block and vice versa. ${ }^{48}$

\section{Ischemic alternans}

Alternans rhythm is commonly seen in the ischemic ventricle, often immediately preceding the phase of induction of malignant arrhythmias such as ventricular tachycardia and fibrillation. ${ }^{3-11}$ On the clinical electrocardiogram, alternation of either the ST-segment or the T-wave (or both) has been the most commonly described form of alternans during ischemia. ${ }^{5,6,8}$ Indeed, instrumentation to check for occult T-wave alternation in the clinical electrocardiogram using power spectral analysis is presently being evaluated in an attempt to identify patients at an increased risk of sudden cardiac death (CH 2000 Cardiac Diagnostic System, Cambridge Heart Inc.). ${ }^{8}$ The alternation in the T-wave of the electrocardiogram may be due to an intrinsic beat-to-beat alternation in the action potential duration in the ischemic area, being essentially equivalent to the "primary" alternans ${ }^{47}$ seen in a periodically stimulated isolated ventricular cell ${ }^{25,27,28}$ and in ionic models of space-clamped ventricular membrane..$^{27,42,43}$ Alternatively, the alternans might be due to electrotonic coupling with a nearby area showing 2:1 block (see, e.g., Fig. 10 of Ref. 3 and Fig. 5 of Ref. 4), resulting in "secondary" alternans. ${ }^{47}$ Recent modeling work with the LR model supports the secondary origin of the alternans in ischemia. ${ }^{68}$

There are instances in which alternans first appears during ischemia immediately after a spontaneous or externally elicited premature ventricular contraction ${ }^{5,6,9}$ or following a single intentionally inserted long cycle. ${ }^{9}$ The most obvious possibility to account for this finding is that there is bistability between 1:1 rhythm and primary alternans in the ischemic area, as can occur in maps derived from ionic models. ${ }^{42,59}$ However, since both excitability and intercellular stimulation current are reduced in ischemia, and ischemia flattens the APDRC determined from a premature stimulation protocol, ${ }^{9,69,70}$ one might expect to see the $\{1: 1 \leftrightarrow 2: 1\}$ bistability in ischemic muscle. [Indeed, when agents that flatten the APDRC and suppress alternans are used (e.g., DAM, $\mathrm{Ca}^{++}$-channel blockers), ${ }^{22}$ one might also expect a similar effect. $\left.{ }^{14}\right]$ It is thus possible that when "triggered alternans",9 follows a premature ventricular contraction, the premature stimulus is flipping an area showing marginal 1:1 conduction to $2: 1$ conduction, thus inducing secondary alternans in the surrounding area (or vice versa, abolishing alternans). 5,6,9

In addition, spatially discordant alternans (alternans in anti-phase at two locations), which is more malignant than concordant alternans, ${ }^{10,11,21}$ can be transformed into the latter during ischemia by a premature ventricular contraction (either spontaneous or stimulated), a reduction of the BCL for one cycle, or a pause. ${ }^{7}$ Again, it is possible that flips between 1:1 and 2:1 rhythms might somehow be involved: e.g., if the out-of phase alternans seen during discordant alternans is secondary (i.e., each of the two types of alternans is associated with its own area of 2:1 block), flipping one area of 2:1 block to $1: 1$ rhythm would result in a conversion from discordant to concordant alternans. However, a simple model using the concept of a critical phase-reversal point during primary alternans shows how a suitably timed extrastimulus can flip concordant to discordant alternans or vice versa, ${ }^{28}$ suggesting bistability between two different spatially organized primary alternans rhythms [see also Fig. 5 of Ref. 16, Fig. 6 of Ref. 39, Fig. 8(b) of Ref. 58, and Figs. 5 and 6(d) of Ref. 59 for coexistence of two different primary alternans rhythms.]

In conclusion, only two forms of bistability of periodic rhythms, $\{1: 1 \leftrightarrow 2: 1\}$ and $\{1: 1 \leftrightarrow 1: 0\}$, have been systematically investigated in periodically stimulated cardiac tissue. It is our expectation that other forms of bistability, or even multistability (the simultaneous presence of three or more rhythms), will be found in the future (perhaps even involving reentrant rhythms). In particular, if there is indeed a causal connection between alternans and malignant ventricular arrhythmias in the setting of acute myocardial ischemia, the ability to replace one rhythm with another (or one form of alternans with a less malignant form) by injecting a welltimed stimulus might eventually be of some clinical use. 


\section{ACKNOWLEDGMENTS}

We thank Aoxiang Xu for help with computer programming and Arthur Lo for help with data analysis. The experimental and modeling components of the work described above were supported by research grants to M. Guevara from the Heart and Stroke Foundation of Quebec (1986-1991) and the Medical Research Council of Canada, respectively. Salary support for A.Yehia provided by a team grant from the Fonds pour la Formation de Chercheurs et l'Aide à la Recherche (Québec).

${ }^{1}$ G. J. Anderson, K. Greenspan, and C. Fisch, "Electrophysiologic studies on Wenckebach structures below the atrioventricular junction," Am. J. Cardiol. 30, 232-236 (1972).

${ }^{2}$ N. El-Sherif, R. R. Hope, B. J. Scherlag, and R. Lazzara, "Re-entrant ventricular arrhythmias in the late myocardial infarction period. 2. Patterns of initiation and termination of re-entry," Circulation 55, 702-719 (1977).

${ }^{3}$ E. Downar, M. J. Janse, and D. Durrer, "The effect of acute coronary artery occlusion on subepicardial transmembrane potentials in the intact porcine heart," Circulation 56, 217-224 (1977).

${ }^{4}$ D. L. Carson, R. Cardinal, P. Savard, and M. Vermeulen, "Characterisation of unipolar waveform alternation in acutely ischaemic porcine myocardium," Cardiovasc. Res. 20, 521-527 (1986).

${ }^{5}$ H. K. Hellerstein and I. M. Liebow, "Electrical alternation in experimental coronary artery occlusion," Am. J. Physiol. 160, 366-374 (1950).

${ }^{6} \mathrm{~K}$. Kataoka and R. Yoshimura, "Clinical and experimental studies on electrical alternans of ST-segment and T-wave in variant form of angina pectoris (in Japanese)," Respiration \& Circulation 27, 767-776 (1979).

${ }^{7}$ H. Hashimoto, K. Suzuki, and M. Nakashima, "Effects of the ventricular premature beat on the alternation of the repolarization phase in ischemic myocardium during acute coronary occlusion in dogs," J. Electrocardiol. 17, 229-238 (1984)

${ }^{8}$ D. S. Rosenbaum, P. Albrecht, and R. J. Cohen, "Predicting sudden cardiac death from $T$ wave alternans of the surface electrocardiogram: promise and pitfalls," J. Cardiovasc. Electrophysiol. 7, 1095-1111 (1996).

${ }^{9}$ S. G. Dilly and M. J. Lab, "Electrophysiological alternans and restitution during acute regional ischaemia in myocardium of anaesthetized pig," J. Physiol. (London) 402, 315-333 (1988).

${ }^{10} \mathrm{H}$. Tachibana, I. Kubota, M. Yamaki, T. Watanabe, and H. Tomoike, "Discordant S-T alternans contributes to formation of reentry: a possible mechanism of reperfusion arrhythmia," Am. J. Physiol. 275, H116-H121 (1998).

${ }^{11}$ T. Konta, K. Ikeda, M. Yamaki, K. Nakamura, K. Honma, I. Kubota, and S. Yasui, "Significance of discordant ST alternans in ventricular fibrillation," Circulation 82, 2185-2189 (1990).

${ }^{12}$ G. R. Mines, "On dynamic equilibrium in the heart," J. Physiol. (London) 46, 349-383 (1913).

${ }^{13}$ S. D. Moulopoulos, N. Kardaras, and D. A. Sideris, "Stimulus-response relationship in dog ventricle in vivo," Am. J. Physiol. 208, 154-157 (1965).

${ }^{14}$ G. M. Hall, S. Bahar, and D. J. Gauthier, "Prevalence of rate-dependent behaviors in cardiac muscle," Phys. Rev. Lett. 82, 2995-2998 (1999).

${ }^{15}$ B. F. Hoffman and E. E. Suckling, "Effect of heart rate on cardiac membrane potentials and the unipolar electrogram,"' Am. J. Physiol. 179, 123130 (1954).

${ }^{16}$ J. B. Nolasco and R. W. Dahlen, "A graphic method for the study of alternation in cardiac action potentials," J. Appl. Physiol. 25, 191-196 (1968).

${ }^{17}$ J. F. Spear and E. N. Moore, "A comparison of alternation in myocardial action potentials and contractility," Am. J. Physiol. 220, 1708-1716 (1971).

${ }^{18}$ M. R. Boyett and B. R. Jewell, "A study of the factors responsible for rate-dependent shortening of the action potential in mammalian ventricular muscle," J. Physiol. (London) 285, 359-380 (1978).

${ }^{19}$ G. V. Savino, L. Romanelli, D. L. González, O. Piro, and M. E. Valentinuzzi, "Evidence for chaotic behavior in driven ventricles,"' Biophys. J. 56, 273-280 (1989).

${ }^{20}$ M. L. Koller, M. L. Riccio, and R. F. Gilmour, Jr., "Dynamic restitution of action potential duration during electrical alternans and ventricular fibrillation," Am. J. Physiol. 275, H1635-H1642 (1998).
${ }^{21}$ J. M. Pastore, S. D. Girouard, K. R. Laurita, F. G. Akar, and D. S. Rosenbaum, "Mechanism linking T-wave alternans to the genesis of cardiac fibrillation,” Circulation 99, 1385-1394 (1999).

${ }^{22}$ M. L. Riccio, M. L. Koller, and R. F. Gilmour, Jr., "Electrical restitution and spatiotemporal organization during ventricular fibrillation," Circ. Res. 84, 955-963 (1999).

${ }^{23}$ M. Delmar, D. C. Michaels, and J. Jalife, "Slow recovery of excitability and the Wenckebach phenomenon in the single guinea pig ventricular myocyte," Circ. Res. 65, 761-774 (1989).

${ }^{24}$ M. R. Guevara, D. Jeandupeux, F. Alonso, and N. Morissette, "Wenckebach rhythms in isolated ventricular heart cells," in International Conference on Singular Behavior and Nonlinear Dynamics, Vol. 2, edited by St. Pnevmatikos, T. Bountis, and Sp. Pnevmatikos (World Scientific, Singapore, 1989), pp. 629-642.

${ }^{25}$ J. Hescheler and R. Speicher, "Regular and chaotic behaviour of cardiac cells stimulated at frequencies between 2 and $20 \mathrm{~Hz}$,' Eur. Biophys. J. 17, 273-280 (1989).

${ }^{26}$ A. R. Yehia, A. Shrier, K. C.-L. Lo, and M. R. Guevara, "Transient outward current contributes to Wenckebach-like rhythms in isolated rabbit ventricular cells," Am. J. Physiol. 273, H1-H11 (1997).

${ }^{27}$ M. R. Guevara, F. Alonso, D. Jeandupeux, and A. C. G. van Ginneken, "Alternans in periodically stimulated isolated ventricular myocytes: Experiment and model," in Cell to Cell Signalling: From Experiments to Theoretical Models, edited by A. Goldbeter (Harcourt Brace Jovanovich, London, 1989), pp. 551-563.

${ }^{28}$ D. S. Rubenstein and S. L. Lipsius, "Premature beats elicit a phase reversal of mechanoelectrical alternans in cat ventricular myocytes: A possible mechanism for reentrant arrhythmias," Circulation 91, 201-214 (1995).

${ }^{29}$ C.-h. Luo and Y. Rudy, "A model of the ventricular cardiac action potential. Depolarization, repolarization and their interaction," Circ. Res. 68, 1501-1526 (1991).

${ }^{30}$ J. W. Moore and F. Ramon, "On numerical integration of the Hodgkin and Huxley equations for a membrane action potential," J. Theor. Biol. 45, 249-273 (1974).

${ }^{31}$ A. Xu and M. R. Guevara, "Two forms of spiral-wave reentry in an ionic model of ischemic ventricular myocardium," Chaos 8, 157-174 (1998).

${ }^{32}$ M. R. Guevara, Chaotic Cardiac Dynamics, Doctoral thesis, McGill University, Montreal, 1984

${ }^{33}$ M. R. Guevara, A. Shrier, and L. Glass, "Chaotic and complex cardiac rhythms," in Cardiac Electrophysiology: From Cell to Bedside, edited by D. P. Zipes and J. Jalife, 1st ed. (W. B. Saunders, Philadelphia, 1990), pp. 192-201.

${ }^{34}$ B. Belles, C. O. Malécot, J. Hescheler, and W. Trautwein, " 'Run-down' of the $\mathrm{Ca}$ current during long whole-cell recordings in guinea pig heart cells: Role of phosphorylation and intracellular calcium," Pflügers Arch. Ges. Physiol. Menschen Tiere 411, 353-360 (1988).

${ }^{35}$ M. R. Guevara, G. Ward, A. Shrier, and L. Glass, "Electrical alternans and period-doubling bifurcations," Computers in Cardiology (IEEE Comp. Soc. Press, Silver Spring, MD, 1984), pp. 167-170.

${ }^{36} \mathrm{E}$. Carmeliet, "Influence du rythme sur la duree du potential d'action ventriculaire cardiaque," Arch. Int. Physiol. Biochim. 63, 222-232 (1955).

${ }^{37}$ A. Varró, P. P. Nánási, and D. A. Lathrop, "Voltage-clamp characteristics of ventricular myocytes in rabbit," Cardioscience 2, 233-243 (1991).

${ }^{38}$ N. C. Janvier, S. O. McMorn, S. M. Harrison, P. Taggart, and M. R. Boyett, "The role of $\mathrm{Na}^{+}-\mathrm{Ca}^{2+}$ exchange current in electrical restitution in ferret ventricular cells," J. Physiol. (London) 504, 301-314 (1997).

${ }^{39}$ Y. Mahler and S. Rogel, "Interrelation between restitution time-constant and alternating myocardial contractility in dogs," Clin. Sci. 39, 625-639 (1970).

${ }^{40}$ R. M. Gulrajani, "Computer simulation of action potential duration changes in cardiac tissue," Computers in Cardiology (IEEE Comp. Soc. Press, Washington, DC, 1987), pp. 629-632.

${ }^{41}$ N. F. Otani and R. F. Gilmour, Jr., "Memory models for the electrical properties of local cardiac systems," J. Theor. Biol. 187, 409-436 (1997).

${ }^{42}$ A. Vinet, D. R. Chialvo, D. C. Michaels, and J. Jalife, "Nonlinear dynamics of rate-dependent activation in models of single cardiac cells," Circ. Res. 67, 1510-1524 (1990).

${ }^{43} \mathrm{~A}$. Vinet and F. A. Roberge, "Excitability and repolarization in an ionic model of the cardiac cell membrane," J. Theor. Biol. 170, 183-199 (1994)

${ }^{44}$ D. R. Chialvo and J. Jalife, "Non-linear dynamics of cardiac excitation and impulse propagation," Nature (London) 330, 749-752 (1987).

${ }^{45}$ D. R. Chialvo, D. C. Michaels, and J. Jalife, "Supernormal excitability as 
a mechanism of chaotic dynamics of activation in cardiac Purkinje fibers," Circ. Res. 66, 525-545 (1990).

${ }^{46} \mathrm{P}$. Lorente and J. Davidenko, "Hysteresis phenomena in excitable cardiac tissues," Ann. N.Y. Acad. Sci. 591, 109-127 (1990).

${ }^{47}$ M. R. Guevara, "Spatiotemporal patterns of block in an ionic model of cardiac Purkinje fibre," in From Chemical to Biological Organization, edited by M. Markus, S. C. Müller, and G. Nicolis (Springer-Verlag, Berlin, 1988), pp. 273-281.

${ }^{48}$ E. Pruvot, A. De Torrente, G. M. De Ferrari, P. J. Schwartz, and J.-J. Goy, "Two-to-one AV block associated with the congenital long QT syndrome," J. Cardiovasc. Electrophysiol. 10, 108-113 (1999).

${ }^{49}$ D. A. Golod, R. Kumar, and R. W. Joyner, "Determinants of action potential initiation in isolated rabbit atrial and ventricular myocytes," Am. J. Physiol. 274, H1902-H1913 (1998).

${ }^{50}$ J. R. Clay, "Monte Carlo simulation of membrane noise: an analysis of fluctuations in graded excitation of nerve membrane,' J. Theor. Biol. 64, 671-680 (1977).

${ }^{51} \mathrm{G}$. W. Beeler and H. Reuter, "Reconstruction of the action potential of ventricular myocardial fibres," J. Physiol. (London) 268, 177-210 (1977).

${ }^{52}$ J.-P. Drouhard and F. A. Roberge, "A simulation study of the ventricular myocardial action potential," IEEE Trans. Biomed. Eng. BME-29, 494502 (1982).

${ }^{53}$ D. M. Bers, "Species differences and the role of sodium-calcium exchange in cardiac muscle relaxation," Ann. N.Y. Acad. Sci. 639, 375-385 (1991).

${ }^{54}$ D. R. Lemieux, F. A. Roberge, and D. Joly, "Modeling the dynamic features of the electrogenic Na,K pump of cardiac cells," J. Theor. Biol. 154, 335-358 (1992).

${ }^{55}$ S. Dokos, B. G. Celler, and N. H. Lovell, "Modification of DiFrancescoNoble equations to simulate the effects of vagal stimulation on in vivo mammalian sinoatrial node electrical activity," Ann. Biomed. Eng. 21, 321-335 (1993).

${ }^{56}$ S. Guan, Q. Lu, and K. Huang, "A discussion about the DiFrancescoNoble model," J. Theor. Biol. 189, 27-32 (1997).

${ }^{57}$ A. Varghese and G. R. Sell, "A conservation principle and its effect on the formulation of Na-Ca exchanger current in cardiac cells," J. Theor. Biol. 189, 33-40 (1997).

${ }^{58}$ T. J. Lewis and M. R. Guevara, "Chaotic dynamics in an ionic model of the propagated cardiac action potential," J. Theor. Biol. 146, 407-432 (1990).
${ }^{59}$ A. Vinet and F. A. Roberge, "Analysis of an iterative difference equation model of the cardiac cell membrane," J. Theor. Biol. 170, 201-214 (1994).

${ }^{60}$ S. Cukierman and A. Paes de Carvalho, "Frequency-dependent excitability of 'membrane' slow responses of rabbit left atrial trabeculae in the presence of $\mathrm{Ba}^{2+}$ and high $\mathrm{K}^{+}$,", J. Gen. Psychol. 79, 1017-1039 (1982).

${ }^{61}$ P. Lorente, C. Delgado, M. Delmar, D. Henzel, and J. Jalife, "Hysteresis in the excitability of isolated guinea pig ventricular myocytes," Circ. Res. 69, 1301-1315 (1991).

${ }^{62}$ J. Beaumont, D. C. Michaels, M. Delmar, J. Davidenko, and J. Jalife, “A model study of changes in excitability of ventricular muscle cells: Inhibition, facilitation, and hysteresis," Am. J. Physiol. 268, H1181-H1194 (1995).

${ }^{63} \mathrm{~W}$. Trautwein and T. F. McDonald, "Current-voltage relations in ventricular muscle preparations from different species," Pflügers Arch. Ges. Physiol. Menschen Tiere 374, 79-89 (1978).

${ }^{64}$ T. Bando, T. Iwa, T. Misaki, and J. Sakurai, "Development of automatic threshold analyser,' Proceedings of the VIth World Symp. Cardiac Pacing. (PACESYMP, Montreal, 1979), Chap. 28-9.

${ }^{65}$ J. M. Davidenko, M. Delmar, J. Beaumont, D. C. Michaels, P. Lorente, and J. Jalife, "Electrotonic inhibition and active facilitation of excitability in ventricular muscle,' J. Cardiovasc. Electrophysiol. 5, 945-960 (1994).

${ }^{66} \mathrm{~N}$. El-Sherif, B. J. Scherlag, and R. Lazzara, "Pathophysiology of second degree atrioventricular block: A unified hypothesis," Am. J. Cardiol. 35, 421-434 (1975).

${ }^{67}$ N. El-Sherif, B. J. Scherlag, R. Lazzara, R. Hope, D. O. Williams, and P. Samet, "The pathophysiology of tachycardia-dependent paroxysmal atrioventricular block after acute myocardial ischemia. Experimental and clinical observations," Circulation 50, 515-528 (1974).

${ }^{68} \mathrm{H}$. Arce et al. (unpublished results).

${ }^{69}$ P. Taggart, P. M. I. Sutton, M. R. Boyett, M. Lab, and H. Swanton, "Human ventricular action potential duration during short and long cycles. Rapid modulation by ischemia," Circulation 94, 2526-2534 (1996).

${ }^{70}$ R. W. Kurz, X. L. Ren, and M. R. Franz, "Dispersion and delay of electrical restitution in the globally ischaemic heart," Eur. Heart J. 15, 547-554 (1994). 